\title{
Mineralogy and Geochemistry of Ultramafic Rocks from Rachoni Magnesite Mine, Gerakini (Chalkidiki, Northern Greece)
}

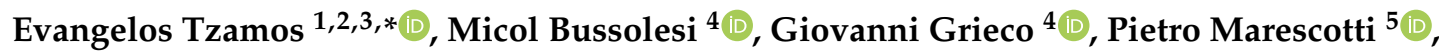 \\ Laura Crispini ${ }^{5}{ }^{\circledR}$, Andreas Kasinos ${ }^{6}$, Niccolò Storni ${ }^{4}$, Konstantinos Simeonidis ${ }^{3}$ \\ and Anastasios Zouboulis ${ }^{1}\left[{ }^{-}\right.$ \\ 1 Department of Chemistry, Aristotle University of Thessaloniki, University Campus, \\ 54124 Thessaloniki, Greece; zoubouli@chem.auth.gr \\ 2 Research \& Development Department, North Aegean Slops SA, 26 Oktovriou str., 42, \\ 54627 Thessaloniki, Greece \\ 3 Ecoresources PC, Giannitson and Santarosa str., 15-17, 54627 Thessaloniki, Greece; \\ simeonidis@ecoresources.gr \\ 4 Department of Earth Sciences, University of Milan, via S. Botticelli 23, 20133 Milan, Italy; \\ micol.bussolesi@unimi.it (M.B.); giovanni.grieco@unimi.it (G.G.); nicostorni@yahoo.it (N.S.) \\ 5 Department for Earth, Environment and Life Sciences, University of Genova, C.so Europa, 26, \\ 16132 Genova, Italy; pietro.marescotti@unige.it (P.M.); laura.crispini@unige.it (L.C.) \\ 6 Grecian Magnesite SA, Gerakini, 63100 Chalkidiki, Greece; a.kasinos@grecianmagnesite.com \\ * Correspondence: tzamos@chem.auth.gr
}

Received: 20 July 2020; Accepted: 19 October 2020; Published: 22 October 2020

\begin{abstract}
The importance of magnesite for the EU economy and industry is very high, making the understanding of their genesis for the exploration for new deposits a priority for the raw materials scientific community. In this direction, the study of the magnesite-hosting ultramafic rocks can be proved very useful. For the present study, ultramafic rock samples were collected from the magnesite ore-hosting ophiolite of the Gerakini mining area (Chalkidiki, Greece) to investigate the consecutive alteration events of the rocks which led to the metallogenesis of the significant magnesite ores of the area. All samples were subjected to a series of analytical methods for the determination of their mineralogical and geochemical characteristics: optical microscopy, XRD, SEM, EMPA, ICP-MS/OES and CIPW normalization. The results of these analyses revealed that the ultramafic rocks of the area have not only all been subjected to serpentinization, but these rocks have also undergone carbonation, silification and clay alteration. The latter events are attributed to the circulation of $\mathrm{CO}_{2}$-rich fluids responsible for the formation of the magnesite ores and locally, the further alteration of the serpentinites into listvenites. The current mineralogy of these rocks was found to be linked to one or more alteration event that took place, thus a significant contribution to the metallo- and petrogenetic history of the Gerakini ophiolite has been made. Furthermore, for the first time in literature, Fe inclusions in olivines from Greece were reported.
\end{abstract}

Keywords: ophiolites; ultramafic; serpentinization; listvenites; Gerakini; magnesite ore

\section{Introduction}

Magnesite ore deposits are closely associated with ophiolitic ultramafic rocks-more specifically dunites and hartzburgites. Magnesite is the main source of magnesium for industry and the raw material needed for the production of various kinds of magnesia $(\mathrm{MgO})$. The large number of applications of magnesia make magnesite one of the most important minerals for industry and the 
economy. In the European Union, magnesite is considered a critical material under the Raw Materials Initiative (RMI). Apart from magnesite and its industrial products, recently many research efforts have been in progress for the valorization of the by-products produced during magnesite mining and processing [1-6]. These mine wastes, namely serpentinized peridotites, are not considered hazardous for the environment or public health; nevertheless, their large volumes make their valorization a critical issue for the achievement of zero waste production and circular economy policies of the European Union. The importance of magnesite ores, their products and mining by-products, make the study of their hosting of ultramafic rocks extremely important for the understanding of their genesis and the exploration of new deposits.

Ultramafic rocks in ophiolite complexes are often subjected to alteration by the infiltration of aqueous fluids [7], resulting in the formation of serpentinites. The infiltration of $\mathrm{CO}_{2}$-rich fluids can produce magnesite through the interaction with a serpentinite or a peridotite. Until today, the source of $\mathrm{CO}_{2}$ remains debatable. Abu-Jaber and Kimberley [8] suggest that $\mathrm{Mg}$ is supplied by the ultramafic host, while the source of carbon remains uncertain. A possible source could be an influx of metamorphic-degassing $\mathrm{CO}_{2}$ and $\mathrm{CH}_{4}$ into groundwater which flows into the ultramafic rocks. $\mathrm{CO}_{2}$-rich water would produce magnesite after interacting with a serpentinite or a peridotite. This model requires a source of carbon in depths greater than $10 \mathrm{~km}\left(\mathrm{~T}>300{ }^{\circ} \mathrm{C}\right)$ for the liberation of volatiles and their mixing with a carbon-poor solution. This mechanism is also supported by Pohl [9], but without excluding the possibility of meteoric water and hydrothermal fluids mixing. Other researchers [10-13] considered the oxidation of organic matter in sedimentary formations, which is then infiltrated by meteoric water into the ultramafic rocks in contact as the main source of $\mathrm{CO}_{2}$-rich fluids. Finally, more recently, Kelemen and Matter [14] and Kelemen et al. [15] supported the exclusively meteoric origin of $\mathrm{CO}_{2}$ and excluded magmatic/metamorphic procedures and the organic matter oxidation as possible sources, with their claim mainly based on the absence of ${ }^{14} \mathrm{C}$ from magnesite ores. Magnesium is provided by the ultramafic host rock by the hydrolysis of Mg-rich minerals. Serpentine, in particular, was demonstrated to be soluble at low $\mathrm{T}$ and $\mathrm{P}$ in pure water [16], and to release $\mathrm{Mg}^{2+}$ ions during weathering [17]. Moreover, an extensive alteration of ultramafic rocks by $\mathrm{CO}_{2}$-rich fluids, can result in "listvenitization", leading to the replacement of the ultramafic protolith by a quartz-carbonate rock denominated listvenite [18]. According to Tsirampides and Filippidis [19], the indicative magnesite ore deposits of Greece are almost 300,000 tons with their value being estimated at $\sim$ EUR 10 billion. Exploitable magnesite deposits in Greece are found mainly in two areas:

- Chalkidiki (e.g., Gerakini, Ormilia, Vavdos, Vasilika); and

- $\quad$ Evia island (e.g., Mantoudi, Limni, Troupi, Petisounas, Afrati, Papades)

Other magnesite occurrences in Greece include the areas of Gomati and Nea Roda (Chalkidiki), Nigrita, Kozani, Grevena, Atalanti, Ermioni and Lesvos. In this work, new insights into the mineralogy and geochemistry of the magnesite-hosting ultramafic rocks of the Gerakini area are presented.

\section{Geologic Setting}

The magnesite deposits of the Chalkidiki (Northern Greece) area are hosted within ophiolitic massifs. According to Kaufmann et al. [20], the Chalkidiki ophiolites are placed into the Circum Rhodope Belt (CRB). The Circum Rhodope Belt sensu stricto comprises low-grade metamorphosed Triassic and Jurassic sedimentary rocks fringing the high-grade metamorphic rocks of the Serbo-Macedonian and Rhodope massifs in northern Greece. Main outcrops occur in the easternmost part of the Vardar suture zone in the Chalkidiki peninsula (Melissochori Formation; formerly Svoula flysch) and in Thrace (Makri unit and Melia Formation) [21].

The tectonostratigraphic relationship between the CRB and the high-grade metamorphics has not yet been clarified: initial research works claimed that the CRB represents the original Mesozoic stratigraphic cover of the Serbo-Macedonian crystalline basement, whereas more recently, the existence of two distinct greenschist facies metamorphic events is proposed [21]. 
The Circum Rhodope Belt is further divided into three geologic formations: Nteve Koran-Doubia Formation, Melissochori-Cholomontas Formation and the Aspri Vrisi-Chortiatis Formation. Ophiolitic massifs hosting magnesite deposits are found excessively in the Aspri Vrisi-Chortiatis Formation, i.e., the ophiolites of Vavdos and Gerakini-Ormilia areas. This ophiolitic complex is a part of the Eastern Ophiolite Belt of Greece.

The Gerakini-Ormilia ophiolite mainly consists of ultramafic and mafic rocks. The lower part of the ophiolite is dominated by serpentinized harzburgites and dunites which are locally intruded by pyroxenitic and gabbroic veins. These peridotites host the stockwork-type magnesite ores which are currently exploited. Additionally, locally some chromite bodies are found which were exploited in the past. Moreover, near faults, massifs of listvenites are preset, hosting vein-type magnesite ores. The upper part of the ophiolite is dominated by massive pyroxenites which are also intruded by magmatic veins.

For the present work, several rock samples of serpentinized ultramafic rocks (dunites and harzburgites) were taken from the Rachoni open pit site in Gerakini (Figure 1).

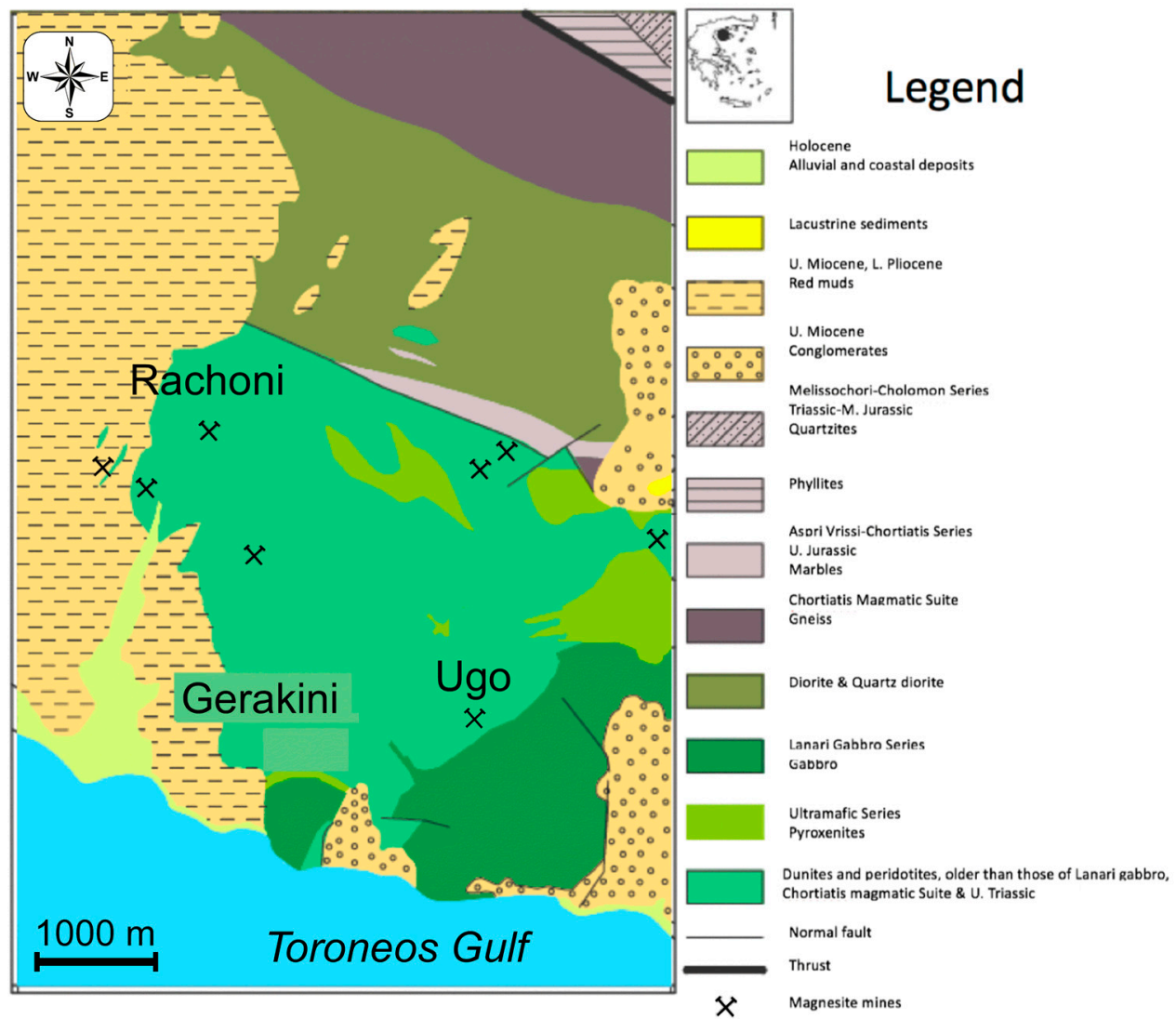

Figure 1. Simplified geological map of the Gerakini mining district [3].

\section{Analytical Methods}

Samples were collected for the present study, including sixteen samples (W1-W16) from the Rachoni magnesite open pit mine in Gerakini. Additionally, one listvenite sample (W17) was collected from the nearby Ugo magnesite open pit mine for comparison. Polished sections and powdered material were prepared for each sample. The mounted polished sections were examined under optical microscope.

The mineralogical characterization of these samples was performed by X-Ray Powder Diffraction (XRPD) using a Bruker D8 Advance diffractometer at the Chemical Engineering Department of the Aristotle University of Thessaloniki. Data were collected at $2 \theta$ from $5^{\circ}$ to $70^{\circ}$, with a scan time of $0.2 \mathrm{~s}$ and increment of 0.02 . The filament $\mathrm{X}$-ray tube of $\mathrm{Cu}$ with a wavelength $1.5418 \AA$ and the detector 
LYNXEYE (1D mode) were used. The identification of the existing mineral phases in XRPD patterns was performed using the PCPDF database while for the quantification, the Rietveld method was applied through the Fullprof software.

Scanning electron microscopy (SEM) images and microprobe analyses (EMPA) were conducted with a JEOL 8200 (JEOL Ltd., Akishima, Japan) equipped with a wavelength dispersive system (WDS) at the Earth Sciences Department of the University of Milan. The microprobe system operated using an accelerating voltage of $15 \mathrm{kV}$, a sample current on brass of $15 \mathrm{nA}$, a counting time of $20 \mathrm{~s}$ on the peaks, and $10 \mathrm{~s}$ on the background. The approximate detection limit was $0.01 \mathrm{wt}$. \% for each element. A series of natural and synthetic standards were used for analysis: $\mathrm{Si}, \mathrm{Ti}, \mathrm{Al}, \mathrm{Cr}, \mathrm{Fe}, \mathrm{Ni}, \mathrm{Mn}, \mathrm{Mg}, \mathrm{Ca}$, $\mathrm{Na}$ and K. EMPA data were corrected for matrix effects by applying the PRZ algorithm included in JEOL software.

The major and trace elements of the whole rock samples were analyzed using a Perkin Elmer ICP-OES (Medtech, Waltham, MA, USA) and a Perkin Elmer Sciex Elan 9000 ICP-MS (Medtech, Waltham, MA, USA), following the $\mathrm{LiBO}_{2} / \mathrm{LiB}_{4} \mathrm{O}_{7}$ fusion and $\mathrm{HNO}_{3}$ digestion of the fused solid sample at the external collaborating laboratories (Bureau Veritas Commodities Canada Ltd., Vancouver, BC, Canada). Based on the chemical analyses of the rock samples, their CIPW normative mineralogy was subsequently calculated.

\section{Results}

\subsection{Petrography and Mineralogy}

Petrographic and mineralogical features are based on XRPD and microscopical observations. Ultramafic rocks comprised of 13 samples of serpentinized peridotites, and one serpentinite sample with plagioclase and a late clay alteration (W3), with three listvenite samples, two from Rachoni mine (W1 and W2) and one from Ugo mine (W17). The three lithologies, which are well distinguished based on their mineralogical assemblage, are reported in Table 1. Microscopic observations on the major mineral phases identified were verified by the XRPD results (Figures 2 and 3).

Table 1. Mineralogical assemblage of the sampled rocks based on optical microscopy study.

\begin{tabular}{ccr}
\hline Lithology & Sample & Mineralogy \\
\hline Listvenite & W1 & quartz, plagioclase $( \pm$ mica, amphibole, dolomite, spinel, chlorite $)$ \\
\hline Listvenite & W2 & quartz, dolomite $( \pm$ calcite, spinel, chlorite $)$ \\
\hline Clayey Serpentinite & W3 & serpentine, magnesite, vermiculite $( \pm$ olivine, amphibole, chlorite, plagioclase $)$ \\
\hline Peridotite & W4 & olivine, orthopyroxene, clinopyroxene, serpentine $( \pm$ spinel, calcite $)$ \\
\hline Peridotite & W5 & olivine, serpentine $( \pm$ orthopyroxene, spinel, amphibole, dolomite $)$ \\
\hline Peridotite & W6 & olivine, serpentine, magnesite $( \pm$ orthopyroxene, spinel, amphibole, chlorite $)$ \\
\hline Peridotite & W7 & olivine, serpentine, pyroxene, magnesite $( \pm$ spinel, dolomite, calcite $)$ \\
\hline Peridotite & W8 & olivine, serpentine, orthopyroxene $( \pm$ spinel $)$ \\
\hline Peridotite & W9 & olivine, serpentine, orthopyroxene $( \pm$ spinel $)$ \\
\hline Peridotite & W10 & olivine, serpentine, orthopyroxene $( \pm$ spinel $)$ \\
\hline Peridotite & W11 & olivine, serpentine, orthopyroxene $( \pm$ spinel $)$ \\
\hline Peridotite & W12 & olivine, serpentine, orthopyroxene $( \pm$ spinel $)$ \\
\hline Peridotite & W13 & olivine, serpentine, orthopyroxene $( \pm$ spinel $)$ \\
\hline Peridotite & W14 & olivine, serpentine, orthopyroxene $( \pm$ spinel $)$ \\
\hline Peridotite & W15 & olivine, serpentine, orthopyroxene $( \pm$ spinel $)$ \\
\hline Peridotite & W16 & olivine, serpentine $( \pm$ spinel, Fe-Mg hydroxide $)$ \\
\hline Listvenite & W17 & quartz, dolomite, magnesite, serpentine $( \pm$ spinel $)$ \\
\hline
\end{tabular}




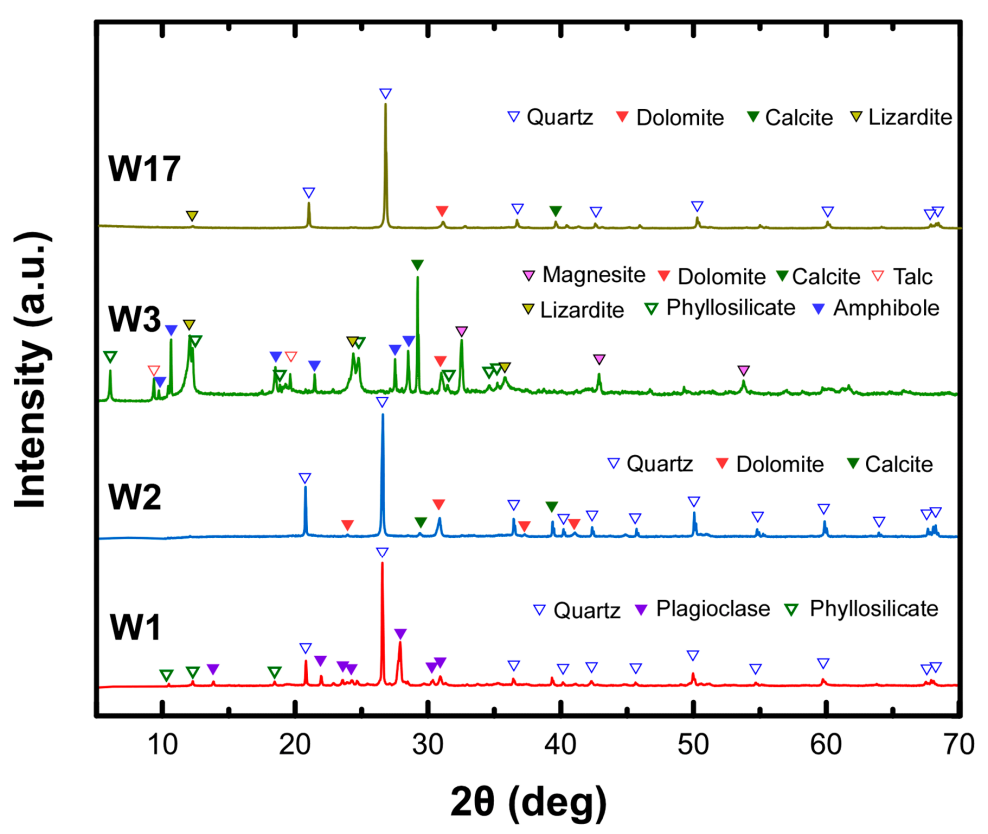

Figure 2. XRPD patterns of listvenite (W1, W2, W17) and clayey serpentinite (W3) samples. The phyllosilicate in sample W1 is mica whereas in sample W3 it is chlorite.

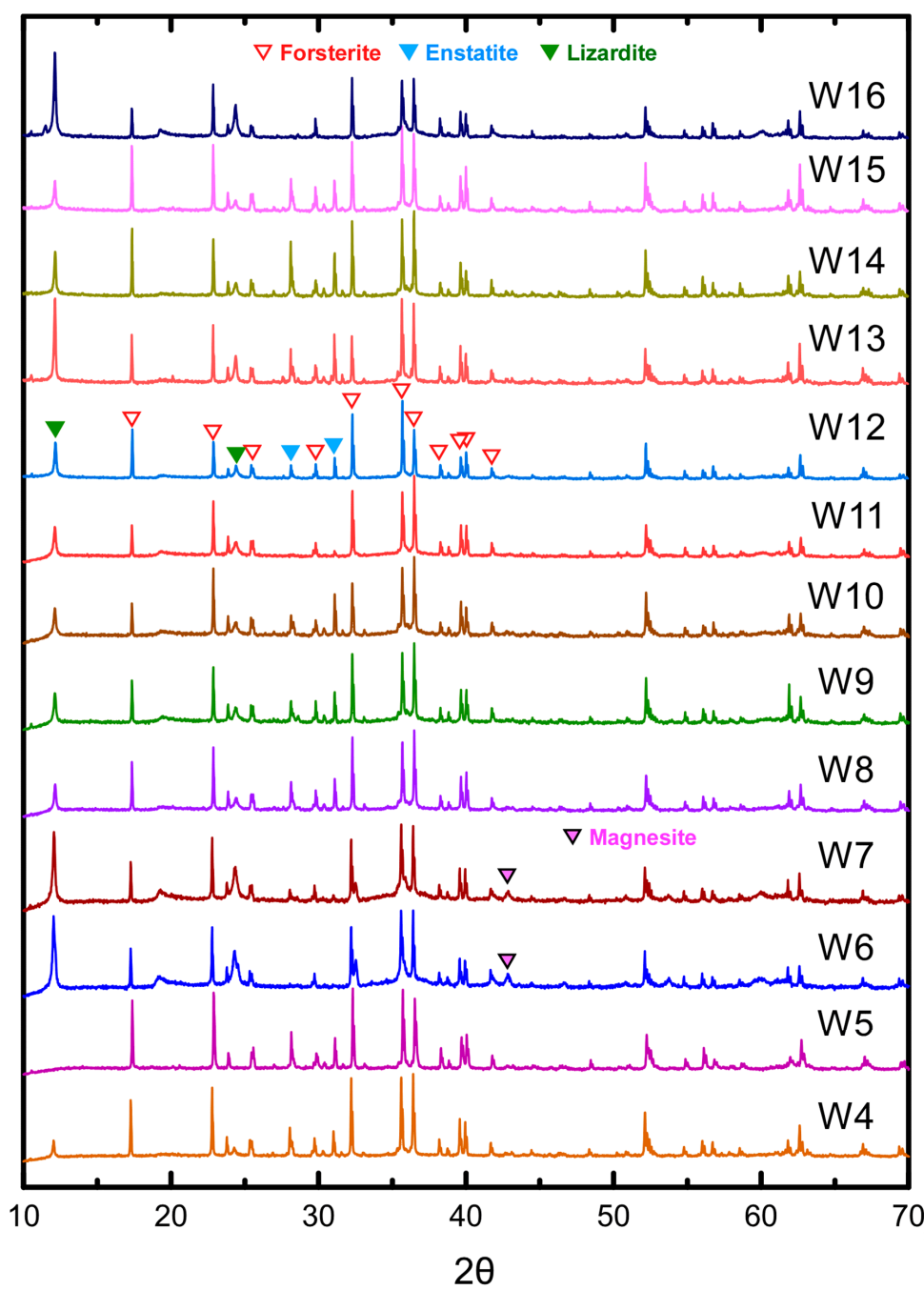

Figure 3. XRPD patterns of peridotite samples. 
Peridotites are characterized by the abundance of olivine and orthopyroxene relicts in a serpentine matrix. Minor and accessory phases are clinopyroxene, chlorite, carbonates, amphiboles and spinels. The mineralogical content of the peridotites, as determined by the Rietveld method, is presented in Figure 4.

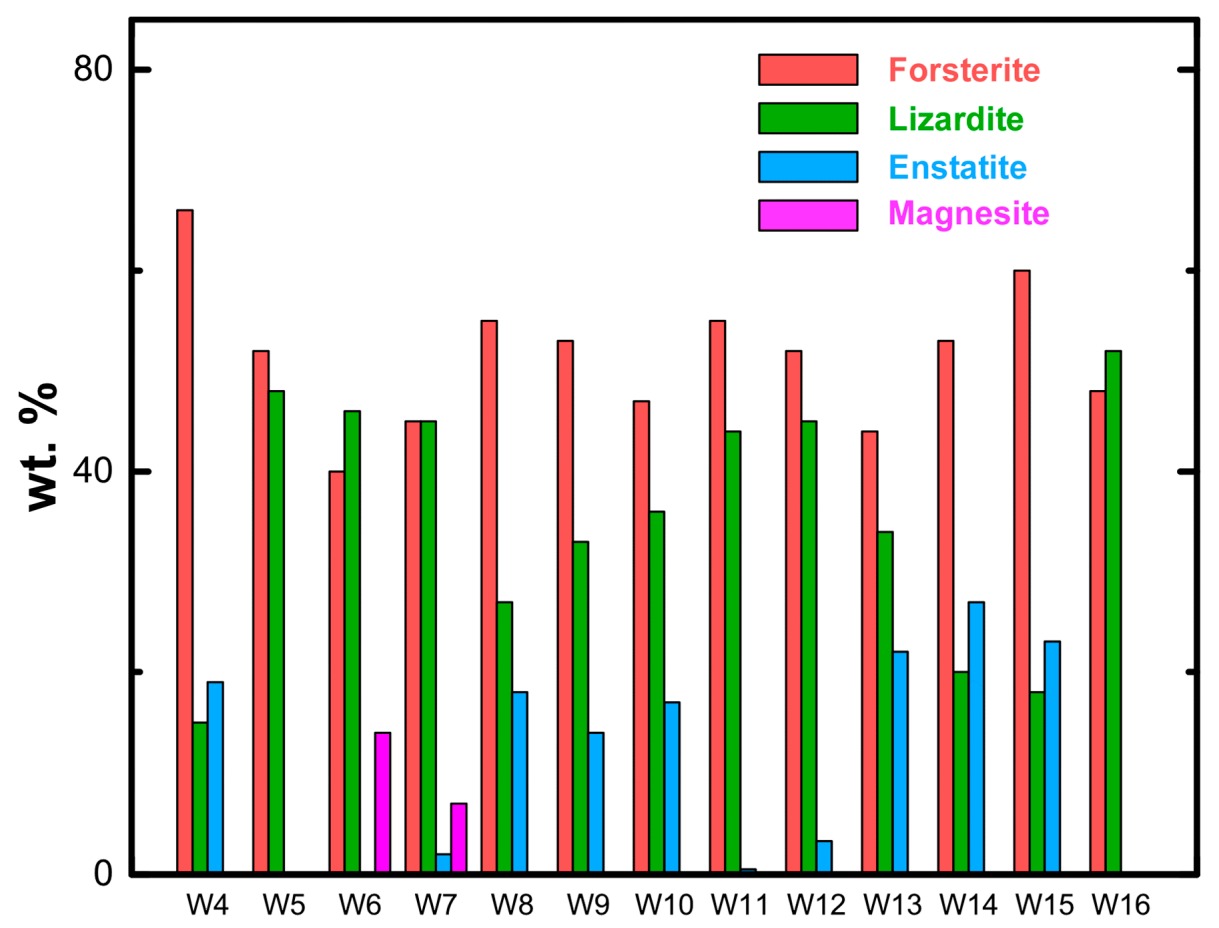

Figure 4. Mineralogical content (wt.\%) of peridotite samples W4-W16.

Olivines occur in roundish and intensely fractured relics within the serpentine matrix with typical mesh texture (Figure 5A-D,F,H). Irregular patches of a Fe-rich olivine are also present in some of the samples (Figure 5B). Orthopyroxenes occur as fractured grains with equigranular shape (Figure 5C,F,J). Serpentine is present partially replacing olivine along rims and as an interstitial phase (Figure 5A-I). Fe-rich serpentine, when present, forms thin irregular rims bordering serpentine grains (Figure 5D,G). The most abundant carbonate phase is magnesite, forming colorless anhedral grains (Figure 5A,E). Three varieties of spinels can be observed: large unaltered chromite grains (Figure 5F,I), altered ferrian chromite grains with a characteristic spongy texture (Figure 5G) and small magnetite grains mainly bordering olivine crystals (Figure 5D,H). Accessory phases are clinopyroxenes, occurring as colorless intensely fractured grains (Figure 5I,J), rare amphiboles (Figure 5G), calcite and dolomite (Figure 5I).

Listvenites, found in association with peridotites, show intense alteration and are crosscut by magnesite veins (Figure 6A). Listvenitized samples show variable degrees of carbonation and silicification and different mineralogical associations. Their recurrent mineralogical association is quartz + carbonates. The most abundant carbonate is dolomite, followed by calcite and minor magnesite. Biotite mica occurs in some samples as a minor listvenite phase. Relict phases, witnesses of the ultramafic protolith are partially spinel (chromite and Fe-chromite), serpentine, chlorite, and amphibole. These minerals are also indicative of two processes predating or co-occurring with listvenitization: serpentinization and chloritization. Quartz is the most abundant phase. It forms aggregates of roundish crystals characterized by wavy extinction (Figure 6B). Plagioclase, when present, forms euhedral crystals, crossed by carbonate-filled fractures (Figure 6C). Dolomite forms euhedral crystals (Figure 6D), from colorless to rosy and slightly pleocroic, with typical polysynthetic geminations that appear as lamellae oriented according to the short diagonals of the rhombohedral cleavage. Serpentine is present either in small veins, as aggregates of pseudomorphic crystals, or bordering quartz crystals 
in Fe-rich variety greenalite (Figure 6D). Opaque phases are mainly $\mathrm{Cr}$-spinels with little to absent Fe-chromitization (Figure 6D).
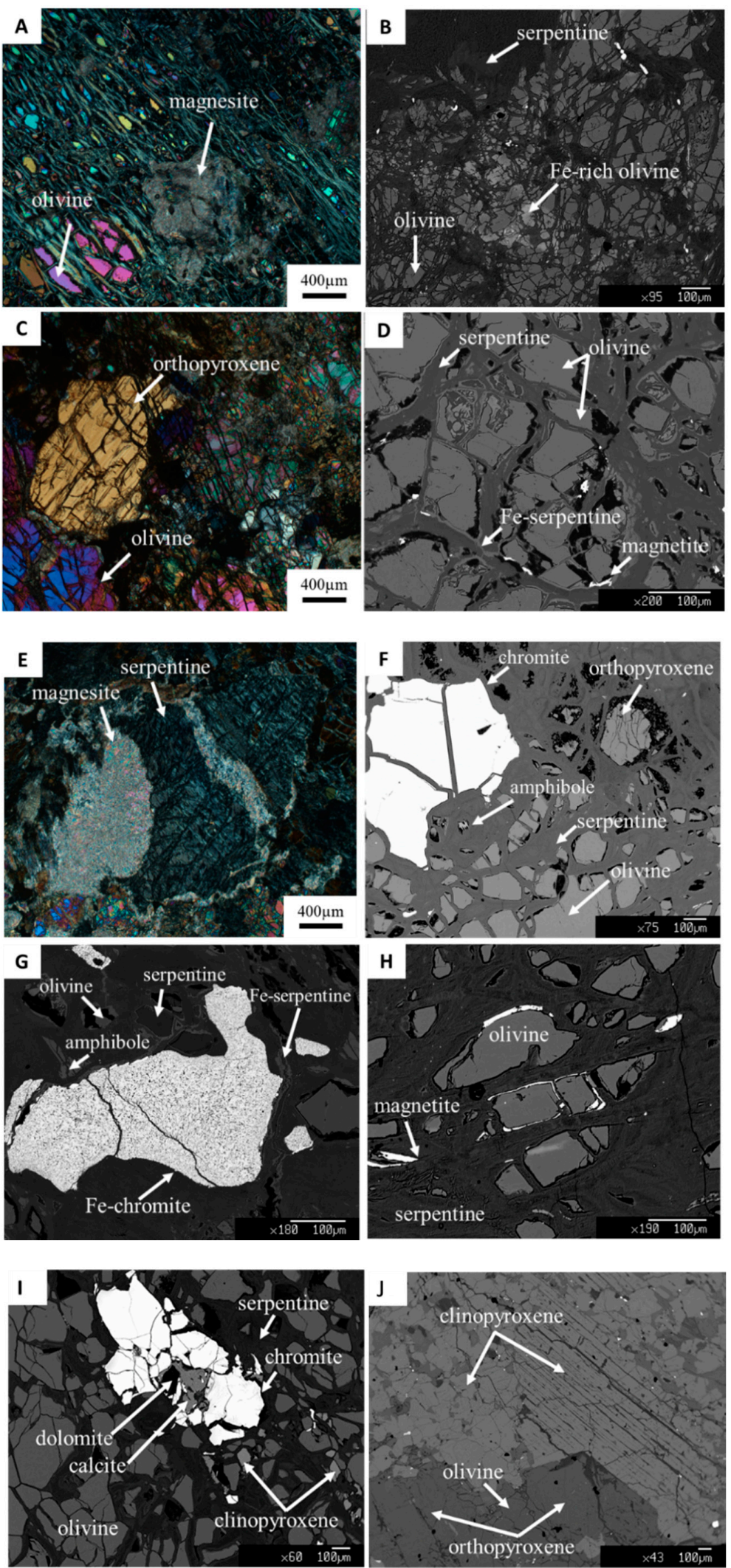

Figure 5. Optical microscopy (crossed polars; $(\mathbf{A}, \mathbf{C}, \mathbf{E})$ ) and back-scattered images $(\mathbf{B}, \mathbf{D}, \mathbf{F}-\mathbf{J})$ of serpentinized peridotites from the Rachoni mine (from samples W6, W13, W14, W15, W16). 

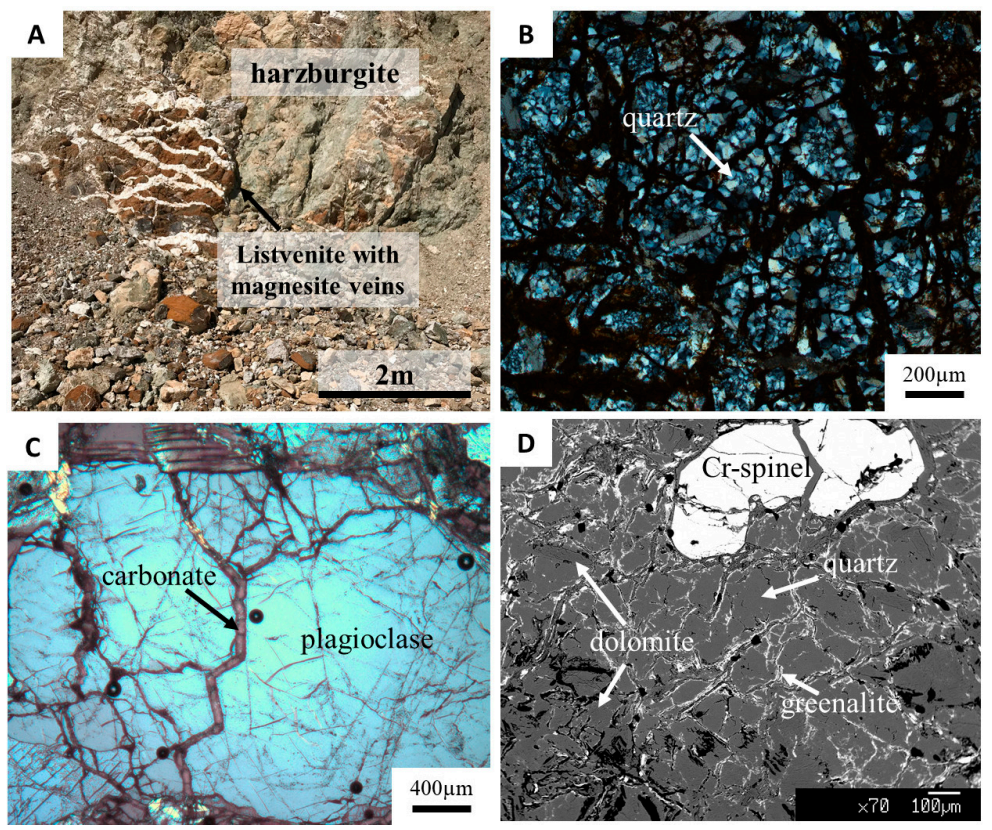

Figure 6. Optical characteristics of listvenites: (A) outcrop picture of a listvenite from the Rachoni mine; (B,C) the optical microscopy (crossed polars) images of listvenites from an Rachoni open pit (samples W2 and W1); and (D) back-scattered image of a listvenite from the Ugo (W17) open pit.

Sample W3 is constituted by a serpentine matrix with abundant plagioclase, carbonates and amphiboles. Plagioclase forms euhedral crystals often presenting the characteristic albite polysynthetic twinning (Figure 7A,B,D). Magnesite is the prevalent carbonate and forms colorless anhedral grains (Figure 7A,B). Serpentine is present in the aggregates of pseudomorphic crystals with a lamellar colorless to pale green structure, with low relief and low birefringence (Figure 7A,B). The few primary silicate relicts are mainly represented by rounded and intensely fractured olivine relics (Figure 7C).
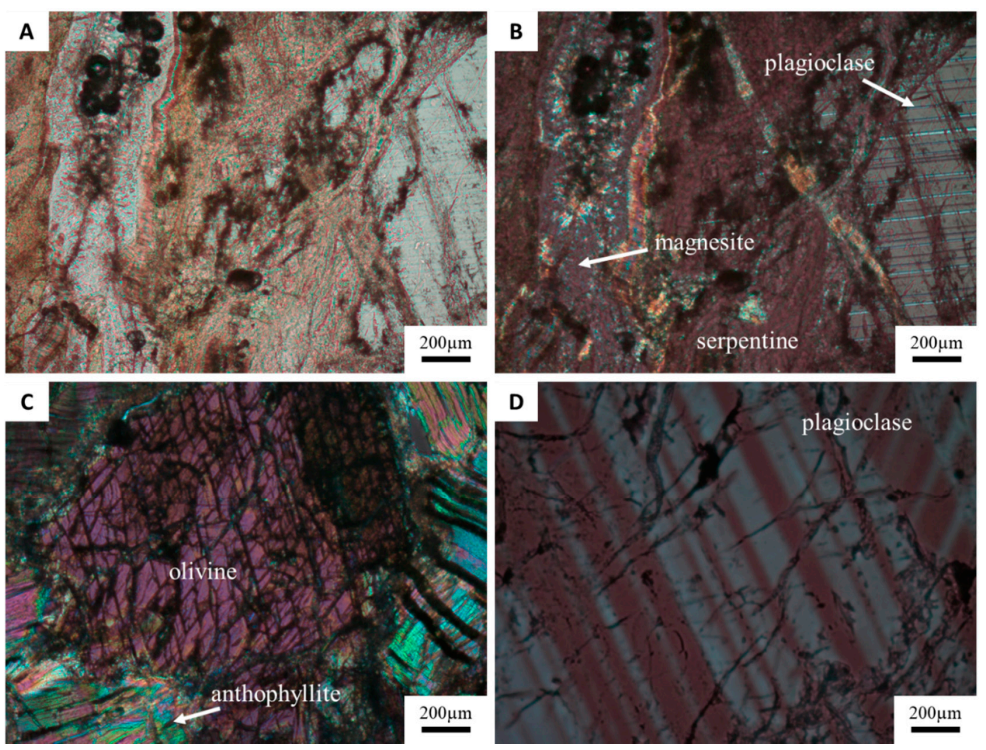

Figure 7. Optical microscopy (plane polarized light; (A); crossed polars; (B-D)) images of a clayey serpentinite with abundant plagioclase (sample W3); (A,B) magnesite vein cutting the serpentine matrix; (C) fractured olivine relic in contact with anthophyllite; and (D) pagioclase with twinning.

\subsection{Bulk-Rock Geochemistry}

Bulk-rock compositions of the samples are reported in Table 2. 
Table 2. Whole rock compositions of the studied samples. Oxides and loss of ignition (LOI) concentration values are presented in wt.\% and trace elements concentrations in ppm.

\begin{tabular}{|c|c|c|c|c|c|c|c|c|c|c|c|c|c|c|c|c|c|}
\hline Lithology & & stvenit & & Serpen & inite & & & & & & ridotites & & & & & & \\
\hline Sample & W1 & W2 & W17 & W3 & W4 & W5 & W6 & W7 & W8 & W9 & W10 & W11 & W12 & W13 & W14 & W15 & W16 \\
\hline $\mathrm{SiO}_{2}$ & 64.92 & 65.31 & 71.99 & 40.63 & 41.80 & 37.22 & 35.02 & 36.63 & 42.48 & 42.94 & 41.92 & 38.37 & 40.08 & 42.99 & 43.55 & 42.41 & 39.22 \\
\hline $\mathrm{Al}_{2} \mathrm{O}_{3}$ & 10.74 & 1.23 & 0.13 & 2.68 & 0.55 & 0.32 & 0.24 & 0.34 & 0.55 & 0.55 & 0.62 & 0.22 & 0.34 & 0.65 & 0.62 & 0.74 & 0.30 \\
\hline $\mathrm{Fe}_{2} \mathrm{O}_{3}(\mathrm{~T})$ & 1.88 & 7.23 & 5.76 & 5.76 & 8.34 & 8.50 & 7.90 & 8.18 & 8.62 & 8.65 & 8.41 & 8.23 & 8.92 & 8.69 & 8.75 & 8.51 & 8.50 \\
\hline $\mathrm{MnO}$ & 0.04 & 0.11 & 0.07 & 0.11 & 0.12 & 0.15 & 0.11 & 0.12 & 0.12 & 0.12 & 0.12 & 0.11 & 0.12 & 0.12 & 0.12 & 0.12 & 0.11 \\
\hline $\mathrm{MgO}$ & 8.42 & 5.82 & 7.24 & 33.32 & 45.16 & 43.86 & 41.91 & 42.21 & 41.65 & 40.68 & 40.94 & 43.38 & 44.03 & 41.94 & 42.32 & 43.41 & 43.12 \\
\hline $\mathrm{CaO}$ & 3.95 & 7.44 & 3.98 & 1.52 & 0.54 & 0.47 & 0.30 & 0.47 & 0.83 & 0.68 & 0.75 & 0.26 & 0.35 & 1.36 & 0.76 & 0.86 & 0.35 \\
\hline $\mathrm{Na}_{2} \mathrm{O}$ & 2.95 & 0.05 & 0.02 & 0.10 & $<0.01$ & $<0.01$ & $<0.01$ & $<0.01$ & 0.05 & 0.05 & 0.04 & 0.05 & 0.06 & 0.07 & 0.06 & 0.05 & 0.06 \\
\hline $\mathrm{K}_{2} \mathrm{O}$ & 0.38 & 0.06 & 0.01 & 0.01 & $<0.01$ & $<0.01$ & $<0.01$ & $<0.01$ & $<0.01$ & 0.01 & $<0.01$ & $<0.01$ & $<0.01$ & $<0.01$ & $<0.01$ & $<0.01$ & $<0.01$ \\
\hline $\mathrm{TiO}_{2}$ & 0.08 & 0.05 & $<0.01$ & 0.05 & $<0.01$ & $<0.01$ & $<0.01$ & $<0.01$ & $<0.01$ & $<0.01$ & $<0.01$ & $<0.01$ & $<0.01$ & $<0.01$ & 0.01 & $<0.01$ & 0.01 \\
\hline $\mathrm{P}_{2} \mathrm{O}_{5}$ & 0.02 & 0.04 & 0.02 & 0.02 & 0.01 & 0.01 & $<0.01$ & $<0.01$ & 0.00 & 0.00 & $<0.001$ & 0.00 & 0.01 & 0.01 & 0.01 & 0.01 & 0.01 \\
\hline $\mathrm{SO}_{3}$ & na & na & 0.01 & na & na & na & na & na & 0.02 & 0.02 & 0.02 & 0.02 & 0.02 & 0.02 & 0.02 & 0.01 & 0.06 \\
\hline $\mathrm{Cr}_{2} \mathrm{O}_{3}$ & na & na & na & na & na & na & na & na & 0.44 & 0.44 & 0.45 & 0.43 & na & na & na & na & na \\
\hline LOI & 6.87 & 12.47 & 9.75 & 15.96 & 3.50 & 9.49 & 14.43 & 12.35 & 4.96 & 5.81 & 6.12 & 8.52 & 5.63 & 3.64 & 3.04 & 3.26 & 7.84 \\
\hline Total & 100.20 & 99.80 & 98.98 & 100.15 & 100.02 & 100.02 & 99.91 & 100.30 & 99.72 & 99.94 & 99.39 & 99.59 & 99.56 & 99.48 & 99.25 & 99.37 & 99.57 \\
\hline $\mathrm{Sc}$ & 4.0 & 6.0 & 2.7 & 6.0 & 8.0 & 5.0 & 5.0 & 7.0 & 10.0 & 9.8 & 9.6 & 4.6 & 6.6 & 11.1 & 10.3 & 8.7 & 6.3 \\
\hline $\mathrm{Be}$ & 2.0 & $<1$ & 0.4 & $<1$ & $<1$ & $<1$ & $<1$ & $<1$ & $<0.2$ & 0.6 & $<0.2$ & $<0.2$ & 0.2 & 0.2 & 0.4 & 0.2 & $<0.2$ \\
\hline V & 15.0 & 51.0 & 123.0 & 17.0 & 30.0 & 23.0 & 16.0 & 26.0 & 39.0 & 39.4 & 41.7 & 15.8 & 25.0 & 45.3 & 43.6 & 37.8 & 21.8 \\
\hline $\mathrm{Cr}$ & 570 & 3990 & 2270 & 1720 & 3060 & 4640 & 3090 & 2870 & 3180 & 3150 & 3150 & 3020 & 3140 & 2870 & 3110 & 2820 & 2930 \\
\hline Co & 18.0 & 91.0 & 80.6 & 61.0 & 116.0 & 122.0 & 112.0 & 110.0 & 113.0 & 113.0 & 110.0 & 119.0 & 123.0 & 113.0 & 114.0 & 113.0 & 116.0 \\
\hline $\mathrm{Ni}$ & 410 & 1450 & 1270 & 1400 & 2460 & 2500 & 2410 & 2220 & 2330 & 2390 & 2330 & 2570 & 2490 & 2330 & 2350 & 2370 & 2400 \\
\hline $\mathrm{Cu}$ & 20 & 10 & 4 & $<10$ & 10 & 10 & 20 & $<10$ & 14 & 10 & 14 & 4 & 6 & 22 & 12 & $<2$ & 4 \\
\hline $\mathrm{Zn}$ & $<30$ & 50 & 35 & 60 & 40 & 50 & 50 & 40 & 50 & 50 & 50 & 45 & 50 & 45 & 45 & 45 & 45 \\
\hline $\mathrm{Ga}$ & 10.0 & 2.0 & 0.3 & 4.0 & 1.0 & 1.0 & $<1$ & 1.0 & 0.7 & 0.7 & 0.7 & 0.4 & 0.4 & 0.7 & 0.7 & 0.5 & 0.4 \\
\hline $\mathrm{Ge}$ & 0.7 & 0.8 & 0.7 & 1.3 & 0.7 & 1.0 & 0.7 & 0.8 & 0.9 & 0.8 & 0.8 & 0.6 & 0.7 & 0.8 & 0.8 & 0.8 & 0.7 \\
\hline As & $<5$ & $<5$ & 3.2 & $<5$ & $<5$ & $<5$ & $<5$ & $<5$ & 0.6 & 0.8 & 0.6 & 0.6 & $<0.2$ & $<0.2$ & $<0.2$ & $<0.2$ & $<0.2$ \\
\hline $\mathrm{Rb}$ & 8.0 & 3.0 & 2.3 & 2.0 & $<1$ & $<1$ & $<1$ & $<1$ & 0.6 & 0.9 & 0.7 & 0.4 & 0.3 & 0.3 & 0.4 & $<0.05$ & $<0.05$ \\
\hline $\mathrm{Sr}$ & 238.0 & 81.0 & 20.9 & 31.0 & $<2$ & $<2$ & $<2$ & $<2$ & 3.2 & 3.8 & 2.5 & 3.1 & 1.7 & 2.3 & 2.5 & 1.1 & 0.8 \\
\hline $\mathrm{Y}$ & 8.8 & 2.5 & 0.1 & 5.6 & $<0.5$ & $<0.5$ & $<0.5$ & $<0.5$ & 0.1 & 0.1 & 0.1 & 0.1 & $<0.02$ & 0.1 & 0.1 & $<0.02$ & $<0.02$ \\
\hline $\mathrm{Zr}$ & 69.0 & 17.0 & $<0.5$ & 22.0 & 2.0 & 5.0 & 2.0 & $<1$ & $<0.5$ & $<0.5$ & 1.0 & $<0.5$ & $<0.5$ & $<0.5$ & $<0.5$ & $<0.5$ & $<0.5$ \\
\hline $\mathrm{Nb}$ & 6.5 & 0.2 & 0.1 & 3.0 & $<0.2$ & $<0.2$ & $<0.2$ & $<0.2$ & 0.6 & 0.1 & $<0.01$ & 0.1 & $<0.01$ & $<0.01$ & 0.0 & $<0.01$ & $<0.01$ \\
\hline
\end{tabular}


Table 2. Cont

\begin{tabular}{|c|c|c|c|c|c|c|c|c|c|c|c|c|c|c|c|c|c|}
\hline Lithology & & istvenit & & Serpe & tinite & & & & & & ridotites & & & & & & \\
\hline Sample & W1 & W2 & W17 & W3 & W4 & W5 & W6 & W7 & W8 & W9 & W10 & W11 & W12 & W13 & W14 & W15 & W16 \\
\hline Mo & 4.0 & 6.0 & 4.4 & $<2$ & 4.0 & $<2$ & $<2$ & $<2$ & 0.8 & 0.8 & 0.8 & 1.0 & 1.6 & 1.6 & 2.0 & 2.2 & 1.6 \\
\hline $\mathrm{Ag}$ & $<0.5$ & $<0.5$ & $<0.1$ & $<0.5$ & $<0.5$ & $<0.5$ & $<0.5$ & $<0.5$ & $<0.1$ & $<0.1$ & $<0.1$ & $<0.1$ & $<0.1$ & $<0.1$ & $<0.1$ & $<0.1$ & $<0.1$ \\
\hline In & $<0.1$ & $<0.1$ & $<0.05$ & $<0.1$ & $<0.1$ & $<0.1$ & $<0.1$ & $<0.1$ & $<0.05$ & $<0.05$ & $<0.05$ & $<0.05$ & $<0.05$ & $<0.05$ & $<0.05$ & $<0.05$ & $<0.05$ \\
\hline Sn & 3.0 & $<1$ & $<0.2$ & $<1$ & $<1$ & 1.0 & 1.0 & $<1$ & 0.8 & 0.8 & 0.4 & 0.8 & $<0.2$ & $<0.2$ & $<0.2$ & $<0.2$ & $<0.2$ \\
\hline $\mathrm{Sb}$ & $<0.2$ & 0.2 & $<0.1$ & $<0.2$ & $<0.2$ & $<0.2$ & $<0.2$ & $<0.2$ & $<0.1$ & 0.1 & 0.1 & 0.2 & $<0.1$ & $<0.1$ & $<0.1$ & $<0.1$ & $<0.1$ \\
\hline Cs & 0.8 & 0.3 & 0.3 & 0.3 & $<0.1$ & $<0.1$ & $<0.1$ & $<0.1$ & 0.2 & 0.3 & 0.1 & 0.1 & 0.0 & 0.1 & 0.1 & $<0.01$ & $<0.01$ \\
\hline $\mathrm{Ba}$ & 134.0 & 36.0 & 13.0 & 14.0 & 5.0 & 6.0 & 6.0 & 5.0 & $<0.5$ & 5.0 & 1.0 & 0.5 & 1.0 & 1.5 & 1.0 & $<0.5$ & $<0.5$ \\
\hline $\mathrm{La}$ & 34.8 & 2.9 & 0.1 & 14.9 & $<0.05$ & $<0.05$ & $<0.05$ & $<0.05$ & $<0.05$ & 0.1 & $<0.05$ & $<0.05$ & $<0.01$ & $<0.01$ & $<0.01$ & $<0.01$ & $<0.01$ \\
\hline $\mathrm{Ce}$ & 76.8 & 4.9 & $<0.05$ & 27.6 & $<0.05$ & $<0.05$ & $<0.05$ & $<0.05$ & $<0.05$ & $<0.02$ & $<0.02$ & $<0.02$ & $<0.02$ & $<0.02$ & $<0.02$ & $<0.02$ & $<0.02$ \\
\hline $\mathrm{Pr}$ & 7.7 & 0.6 & $<0.01$ & 2.8 & $<0.01$ & $<0.01$ & $<0.01$ & $<0.01$ & $<0.01$ & $<0.01$ & $<0.01$ & $<0.01$ & $<0.01$ & $<0.01$ & $<0.01$ & $<0.01$ & $<0.01$ \\
\hline $\mathrm{Nd}$ & 26.1 & 2.4 & $<0.05$ & 9.6 & $<0.05$ & $<0.05$ & $<0.05$ & $<0.05$ & $<0.01$ & $<0.01$ & $<0.01$ & $<0.01$ & $<0.01$ & $<0.01$ & $<0.01$ & $<0.01$ & $<0.01$ \\
\hline $\mathrm{Sm}$ & 4.5 & 0.5 & $<0.01$ & 1.7 & $<0.01$ & $<0.01$ & $<0.01$ & $<0.01$ & $<0.01$ & $<0.01$ & $<0.01$ & $<0.01$ & $<0.01$ & $<0.01$ & $<0.01$ & $<0.01$ & $<0.01$ \\
\hline $\mathrm{Eu}$ & 0.9 & 0.1 & $<0.05$ & 0.2 & $<0.005$ & $<0.005$ & $<0.005$ & $<0.005$ & $<0.01$ & $<0.01$ & $<0.01$ & $<0.01$ & $<0.01$ & $<0.01$ & $<0.01$ & $<0.01$ & $<0.01$ \\
\hline $\mathrm{Gd}$ & 2.8 & 0.4 & $<0.01$ & 1.0 & $<0.01$ & $<0.01$ & $<0.01$ & $<0.01$ & $<0.01$ & $<0.01$ & $<0.01$ & $<0.01$ & $<0.01$ & $<0.01$ & $<0.01$ & $<0.01$ & $<0.01$ \\
\hline $\mathrm{Tb}$ & 0.3 & 0.1 & $<0.01$ & 0.2 & $<0.01$ & $<0.01$ & $<0.01$ & $<0.01$ & $<0.01$ & $<0.01$ & $<0.01$ & $<0.01$ & $<0.01$ & $<0.01$ & $<0.01$ & $<0.01$ & $<0.01$ \\
\hline Dy & 1.7 & 0.4 & $<0.01$ & 0.9 & $<0.01$ & $<0.01$ & $<0.01$ & $<0.01$ & $<0.01$ & $<0.01$ & $<0.01$ & $<0.01$ & $<0.01$ & $<0.01$ & $<0.01$ & $<0.01$ & $<0.01$ \\
\hline Ho & 0.3 & 0.1 & $<0.01$ & 0.2 & $<0.01$ & $<0.01$ & $<0.01$ & $<0.01$ & $<0.01$ & $<0.01$ & $<0.01$ & $<0.01$ & $<0.01$ & $<0.01$ & $<0.01$ & $<0.01$ & $<0.01$ \\
\hline $\mathrm{Er}$ & 0.8 & 0.3 & $<0.01$ & 0.6 & $<0.01$ & $<0.01$ & $<0.01$ & $<0.01$ & $<0.01$ & $<0.01$ & $<0.01$ & $<0.01$ & $<0.01$ & $<0.01$ & $<0.01$ & $<0.01$ & $<0.01$ \\
\hline $\mathrm{Tm}$ & 0.1 & 0.0 & $<0.01$ & 0.1 & $<0.005$ & $<0.005$ & $<0.005$ & $<0.005$ & $<0.01$ & $<0.01$ & $<0.01$ & $<0.01$ & $<0.01$ & $<0.01$ & $<0.01$ & $<0.01$ & $<0.01$ \\
\hline $\mathrm{Yb}$ & 0.9 & 0.3 & 0.00 & 0.5 & 0.00 & 0.00 & 0.00 & 0.00 & $<0.01$ & 0.00 & 0.00 & $<0.01$ & $<0.01$ & $<0.01$ & 0.00 & 0.00 & $<0.01$ \\
\hline $\mathrm{Lu}$ & 0.2 & 0.0 & $<0.01$ & 0.1 & 0.00 & 0.00 & 0.00 & 0.00 & $<0.01$ & $<0.01$ & $<0.01$ & $<0.01$ & $<0.01$ & $<0.01$ & $<0.01$ & $<0.01$ & $<0.01$ \\
\hline Hf & 2.2 & 0.3 & $<0.01$ & 0.6 & $<0.1$ & 0.1 & $<0.1$ & $<0.1$ & 0.0 & $<0.01$ & $<0.01$ & 0.00 & $<0.01$ & $<0.01$ & $<0.01$ & $<0.01$ & $<0.01$ \\
\hline $\mathrm{Ta}$ & 0.6 & 0.1 & $<0.01$ & 0.2 & 0.0 & 0.0 & 0.0 & 0.0 & 1.2 & 0.3 & 0.1 & 0.1 & $<0.01$ & $<0.01$ & $<0.01$ & $<0.01$ & $<0.01$ \\
\hline W & 0.9 & 2.0 & 2.5 & $<0.5$ & 2.0 & 2.2 & $<0.5$ & 0.5 & 0.5 & 0.5 & $<0.5$ & $<0.5$ & 0.5 & $<0.5$ & 0.5 & 0.5 & 0.5 \\
\hline $\mathrm{Tl}$ & 0.1 & $<0.05$ & $<0.2$ & 0.1 & $<0.05$ & $<0.05$ & $<0.05$ & $<0.05$ & $<0.2$ & $<0.2$ & $<0.2$ & $<0.2$ & $<0.2$ & $<0.2$ & $<0.2$ & $<0.2$ & $<0.2$ \\
\hline $\mathrm{Pb}$ & 15.0 & $<5$ & $<1$ & $<5$ & $<5$ & $<5$ & $<5$ & $<5$ & $<1$ & $<1$ & $<1$ & $<1$ & $<1$ & $<1$ & $<1$ & $<1$ & $<1$ \\
\hline $\mathrm{Bi}$ & $<0.1$ & $<0.1$ & $<0.02$ & $<0.1$ & $<0.1$ & $<0.1$ & $<0.1$ & $<0.1$ & $<0.02$ & $<0.02$ & $<0.02$ & $<0.02$ & $<0.02$ & $<0.02$ & $<0.02$ & $<0.02$ & $<0.02$ \\
\hline Th & 14.8 & 0.8 & $<0.01$ & 5.3 & $<0.05$ & $<0.05$ & $<0.05$ & $<0.05$ & $<0.01$ & 0.0 & $<0.01$ & $<0.01$ & $<0.01$ & $<0.01$ & $<0.01$ & $<0.01$ & $<0.01$ \\
\hline $\mathrm{U}$ & 0.9 & 2.2 & 0.6 & 0.5 & 0.0 & 0.0 & $<0.01$ & $<0.01$ & 0.1 & 0.2 & 0.0 & 0.1 & $<0.01$ & $<0.01$ & $<0.01$ & $<0.01$ & $<0.01$ \\
\hline
\end{tabular}




\subsubsection{Major Elements}

Listvenites are characterized by high variability in major element composition, with $\mathrm{SiO}_{2}$ ranging between 64.9 and $72.0 \mathrm{wt} . \%, \mathrm{Al}_{2} \mathrm{O}_{3}$ from 0.1 to $10.7 \mathrm{wt} . \%, \mathrm{MgO}$ from 5.8 to $8.4 \mathrm{wt} . \%, \mathrm{CaO}$ from 3.9 to $7.4 \mathrm{wt} . \%$, and loss of ignition (LOI) from 6.9 to $12.5 \mathrm{wt} . \%$, depending on the relative abundance of mineral phases. Sample W17, from Ugo mine, has the highest $\mathrm{SiO}_{2}$ and the lowest $\mathrm{Al}_{2} \mathrm{O}_{3}$ contents. Peridotites present lower variability in their major element composition, with $\mathrm{SiO}_{2}$ ranging from 35 to $43.6 \mathrm{wt} . \%, \mathrm{Al}_{2} \mathrm{O}_{3}$ from 0.2 to $0.7 \mathrm{wt} . \%$, and $\mathrm{MgO}$ from 40.7 to $45.2 \mathrm{wt} \%$. Only LOI shows a much wider range of values that appear to be correlated to the degree of serpentinization of the samples. Lowest LOI values for least serpentinized samples is $3 \mathrm{wt} . \%$, whereas the highest value in the strongly serpentinized sample is $14.4 \mathrm{wt} . \%$. Sample W3 has a bulk rock geochemistry comparable to peridotites, at least concerning major elements. $\mathrm{SiO}_{2}$ content is $40.6 \mathrm{wt} . \%, \mathrm{Al}_{2} \mathrm{O}_{3}$ is higher than in peridotites, with a value of $2.7 \mathrm{wt} . \%$, and an $\mathrm{MgO}$ concentration of $33.3 \mathrm{wt} . \%$, intermediate between peridotites and listvenites.

\subsubsection{Trace Elements}

All samples have generally low to very low trace elements contents (mostly below detection limits). All samples have relatively high contents of $\mathrm{V}, \mathrm{Cr}, \mathrm{Co}$, and $\mathrm{Ni}$, due to their ultramafic origin. Listvenites present high levels of $\mathrm{Ga}, \mathrm{Rb}, \mathrm{Sr}, \mathrm{Y}, \mathrm{Zr}, \mathrm{Nb}, \mathrm{Mo}, \mathrm{Ba}, \mathrm{U}$, Th and rare earth elements (REE) with respect to peridotites. Rare earth elements (REE) contents are up to 1000 times higher in listvenites (up to $157.8 \mathrm{ppm}$ ) than in peridotites $(<0.14 \mathrm{ppm})$. REE patterns normalized to primitive mantle are shown in Figure 8. Listvenites W1 and W2 and altered serpentinite W3 present an enrichment in LREE (Light REE) with respect to HREE (Heavy REE), also showing a slight negative Eu anomaly. W17 listvenite has a much lower REE content and less pronounced LREE enrichment, limited to La only. REE profiles could not be plotted for the other samples which were generally below detection limits, something expected for ultramafic ophiolitic rocks.

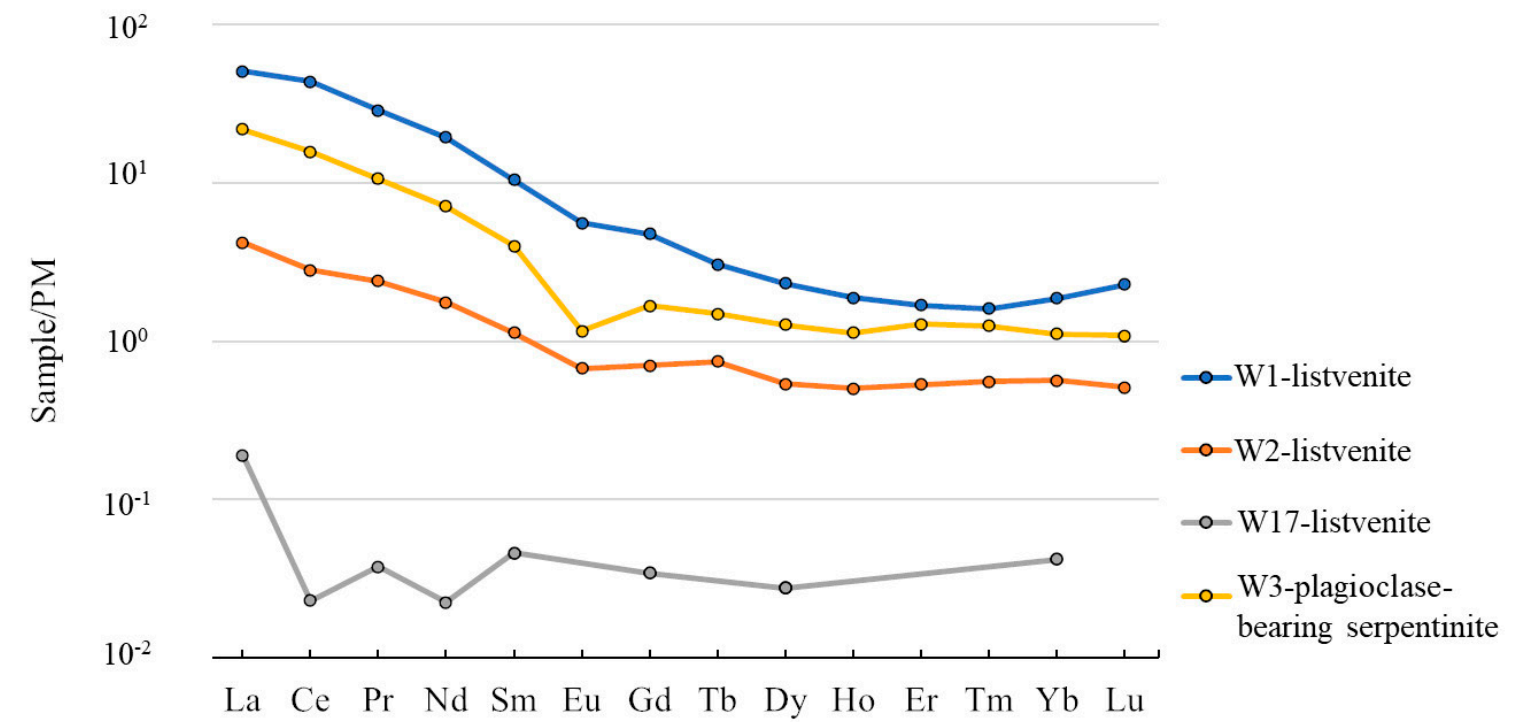

Figure 8. REE patterns normalized to the primitive mantle (McDonough and Sun, 1995) [22] of listvenites and a clayey serpentinite sample W3.

\subsection{CIPW Normative Mineralogy}

Normative mineralogy calculations were performed on partially serpentinized harzburgite rock samples in order to produce an idealized mineralogical association starting from whole rock analyses; the results are shown in Table 3. The application of these calculations was made to classify the studied samples; these samples have undergone various metasomatic events and so the current mineral 
assemblage cannot help under these circumstances. $\mathrm{FeO}$ was calculated from the total $\mathrm{Fe}_{2} \mathrm{O}_{3}$ using the relation: $\mathrm{FeO}=\mathrm{Fe}_{2} \mathrm{O}_{3} \times 0.8998$. In all the samples, olivine is the most abundant mineral, ranging between 62.9 and $86.1 \mathrm{wt} . \%$. Orthopyroxene is the second mineral in order of abundance, ranging between 0.6 and $26.6 \mathrm{wt} . \%$. Other minor components are clinopyroxene (0.7-3.9 wt.\%) and chromite (0.6-1.0 wt.\%). The amount of plagioclase up to $2.2 \mathrm{wt} . \%$ is probably an artifact due to the limitations of the calculating formulae. From the normative mineralogic calculations, four samples (W5, W6, W7 and W11) were classified as dunites, while the remaining samples were harzburgites. However, due to the partial serpentinization of the samples, the differences in olivine and pyroxene abundances could partially be an artifact. As serpentine has roughly the same $\mathrm{SiO}_{2}$ amount as olivine, but less $\mathrm{SiO}_{2}$ amount than pyroxenes, the replacement of pyroxenes by serpentine results in the CIPW calculation in a systematic overestimation of olivine at the highest LOI\%.

\subsection{Mineral Chemistry}

\subsubsection{Serpentinized Peridotites}

Peridotites are characterized by abundant olivine and orthopyroxene relicts in a serpentine matrix. THE mineral chemistry of major and minor phases is reported in Table 4. Olivine is characterized by an average Fo content of 90.8 . A second Fe-rich olivine variety is also present, characterized by lower Fo content (78.4 on average). The most abundant pyroxenes are orthopyroxenes. These are enstatites with an $\mathrm{MgO}$ content ranging from 33.12 and $35.13 \mathrm{wt} . \%$ and $\mathrm{FeO}$ ranging from 5.44 and $6.73 \mathrm{wt} . \%$. Clinopyroxenes are rare and characterized by $\mathrm{MgO}$ content between 15.20 and $18.52 \mathrm{wt} . \%$, FeO content between 1.60 and $2.20 \mathrm{wt} . \%$ and $\mathrm{CaO}$ between 21.92 and $25.52 \mathrm{wt} . \% . \mathrm{Cr}_{2} \mathrm{O}_{3}$ content ranges from 0.30 to $1.04 \mathrm{wt} . \%$. Serpentine partially substitutes primary silicates, and is present in two varieties: one, Fe-poor, replacing olivine; and one Fe-rich, occurring as an interstitial phase. Fe-poor serpentine has $\mathrm{FeO}$ ranging between 1.99 and $8.88 \mathrm{wt} . \%$ whereas the Fe-rich variety has an $\mathrm{FeO}$ content ranging between 14.64 and $23.43 \mathrm{wt} . \%$. Accessory spinels comprise chromite, ferrian chromite and magnetite. Chromites are characterized by an $\mathrm{MgO}$ content ranging from 9.50 to $15.17 \mathrm{wt} . \%, \mathrm{Cr}_{2} \mathrm{O}_{3}$ from 34.53 to $52.22 \mathrm{wt} . \%$, $\mathrm{FeO}$ between 14.47 and $23.24 \mathrm{wt} . \%$, and $\mathrm{Al}_{2} \mathrm{O}_{3}$ between 14.54 and 35.89 wt.\%. Ferrian chromites occur as completely altered grains with a $\mathrm{Cr}_{2} \mathrm{O}_{3}$ content between 34.93 and $51.07 \mathrm{wt} . \%$, an FeOtot content between 42.47 and 55.57 wt. $\%$, an $\mathrm{Al}_{2} \mathrm{O}_{3}$ content between 0.70 and 4.42 wt.\%, and a low $\mathrm{Mg}$ content $(\mathrm{MgO}<3.83 \mathrm{wt} . \%)$. Magnetites are characterized by $\mathrm{Cr}_{2} \mathrm{O}_{3}$ content $<2.28 \mathrm{wt} . \%$. The carbonate phases are, in order of abundance, magnesite, dolomite and calcite. Magnesite is characterized by an $\mathrm{MgO}$ content ranging from 38.21 to $47.88 \%$ and an $\mathrm{FeO}$ content between 0.20 and $26.37 \mathrm{wt} . \%$. Dolomite is characterized by an $\mathrm{MgO}$ content between 21.00 and $24.69 \%$ and $\mathrm{CaO}$ between 24.28 and $32.00 \mathrm{wt} . \%$. Amphibole is very rare and has mainly tremolitic composition. The $\mathrm{MgO}$ content is comprised between 22.57 and $23.54 \mathrm{wt} . \%, \mathrm{CaO}$ between 12.05 and $13.34 \mathrm{wt} . \%$ and $\mathrm{FeO}$ between 1.41 and $2.25 \mathrm{wt} . \%$. Two grains of hornblende were also found.

\subsubsection{Listvenites}

Representative composition of listvenites mineral phases are reported in Table 5. Listvenites are generally fine grained and composed mostly of quartz and carbonates. Among the carbonates, dolomite is the most abundant, whereas calcite is rare. Dolomite in all listvenites is characterized by $\mathrm{CaO}$ and $\mathrm{MgO}$ average contents between 30.8 and 32.5\% and between 21.2 and 22.3\%, respectively. 
Table 3. Normative mineralogy recalculations (CIPW) of the serpentinized peridotites and the classification of the samples into dunites (Dun) and harzburgites (Harz).

\begin{tabular}{|c|c|c|c|c|c|c|c|c|c|c|c|c|c|}
\hline $\begin{array}{c}\text { Rock } \\
\text { Analysis }\end{array}$ & W4 & W5 & W6 & W7 & W8 & W9 & W10 & W11 & W12 & W13 & W14 & W15 & W16 \\
\hline \multicolumn{14}{|c|}{ Normative Minerals wt.\% } \\
\hline Plagioclase & 1.50 & 0.87 & 0.65 & 0.93 & 1.70 & 1.67 & 1.85 & 0.74 & 1.17 & 1.81 & 1.93 & 2.22 & 0.87 \\
\hline Diopside & 0.88 & 1.10 & 0.66 & 1.11 & 2.24 & 1.68 & 1.74 & 0.67 & 0.85 & 3.96 & 1.85 & 1.95 & 0.72 \\
\hline Hypersthene & 13.43 & 1.54 & 0.61 & 3.98 & 21.87 & 26.63 & 22.59 & 7.50 & 9.76 & 20.47 & 23.80 & 16.84 & 10.66 \\
\hline Olivine & 79.93 & 86.13 & 82.85 & 81.18 & 67.69 & 62.85 & 65.84 & 80.98 & 81.32 & 68.73 & 67.79 & 74.33 & 78.57 \\
\hline Ilmenite & 0.02 & 0.00 & 0.00 & 0.00 & 0.00 & 0.00 & 0.00 & 0.00 & 0.00 & 0.00 & 0.02 & 0.00 & 0.02 \\
\hline Magnetite & 0.00 & 0.00 & 0.00 & 0.00 & 0.00 & 0.00 & 0.00 & 0.00 & 0.00 & 0.00 & 0.00 & 0.00 & 0.00 \\
\hline Hematite & 0.00 & 0.00 & 0.00 & 0.00 & 0.00 & 0.00 & 0.00 & 0.00 & 0.00 & 0.00 & 0.00 & 0.00 & 0.00 \\
\hline Chromite & 0.66 & 1.00 & 0.66 & 0.62 & 0.68 & 0.68 & 0.68 & 0.65 & 0.68 & 0.62 & 0.66 & 0.60 & 0.63 \\
\hline Total & 96.42 & 90.64 & 85.43 & 87.82 & 94.18 & 93.57 & 92.70 & 90.54 & 93.78 & 95.59 & 96.05 & 95.94 & 91.47 \\
\hline \multicolumn{14}{|c|}{ Normative Minerals vol $\%$} \\
\hline Plagioclase & 1.87 & 1.16 & 0.92 & 1.27 & 2.20 & 2.17 & 2.42 & 1.00 & 1.53 & 2.28 & 2.45 & 2.80 & 1.15 \\
\hline Diopside & 0.93 & 1.24 & 0.79 & 1.29 & 2.43 & 1.83 & 1.91 & 0.75 & 0.93 & 4.22 & 1.96 & 2.07 & 0.80 \\
\hline Hypersthene & 14.08 & 1.72 & 0.72 & 4.60 & 23.44 & 28.72 & 24.59 & 8.39 & 10.54 & 21.61 & 25.00 & 17.71 & 11.79 \\
\hline Olivine & 82.64 & 95.13 & 97.06 & 92.38 & 71.47 & 66.73 & 70.60 & 89.34 & 86.53 & 71.46 & 70.13 & 77.02 & 85.62 \\
\hline Ilmenite & 0.01 & 0.00 & 0.00 & 0.00 & 0.00 & 0.00 & 0.00 & 0.00 & 0.00 & 0.00 & 0.01 & 0.00 & 0.01 \\
\hline Magnetite & 0.00 & 0.00 & 0.00 & 0.00 & 0.00 & 0.00 & 0.00 & 0.00 & 0.00 & 0.00 & 0.00 & 0.00 & 0.00 \\
\hline Hematite & 0.00 & 0.00 & 0.00 & 0.00 & 0.00 & 0.00 & 0.00 & 0.00 & 0.00 & 0.00 & 0.00 & 0.00 & 0.00 \\
\hline Chromite & 0.45 & 0.72 & 0.51 & 0.46 & 0.47 & 0.47 & 0.48 & 0.47 & 0.47 & 0.42 & 0.45 & 0.41 & 0.45 \\
\hline Total & 99.98 & 99.97 & 100.00 & 100.00 & 100.01 & 100.00 & 100.00 & 99.95 & 100.00 & 99.99 & 100.00 & 100.01 & 99.82 \\
\hline Classification & Harz & Dun & Dun & Dun & Harz & Harz & Harz & Dun & Harz & Harz & Harz & Harz & Harz \\
\hline
\end{tabular}


Table 4. Representative analyses (EMPA) of the peridotite minerals.

\begin{tabular}{|c|c|c|c|c|c|c|c|c|c|c|c|c|c|c|c|c|c|}
\hline \multicolumn{18}{|c|}{ (a) } \\
\hline Mineral & \multicolumn{2}{|c|}{ Olivine } & \multirow{2}{*}{\multicolumn{2}{|c|}{$\begin{array}{c}\text { Fe-rich olivine } \\
\text { W14 }\end{array}$}} & \multicolumn{5}{|c|}{ Serpentine } & \multicolumn{3}{|c|}{ Orthopyroxene } & \multicolumn{3}{|c|}{ Clinopyroxene } & \multicolumn{2}{|c|}{ Tremolite } \\
\hline wt. $\%$ & W6 & W4 & & & W14 & W6 & W5 & W6 & W6 & W16 & W4 & W13 & W16 & W4 & W12 & W6 & W5 \\
\hline $\mathrm{SiO}_{2}$ & 41.53 & 40.69 & \multicolumn{2}{|c|}{39.17} & 39.00 & 39.83 & 41.24 & 34.06 & 32.16 & 57.63 & 57.12 & 56.52 & 53.51 & 54.36 & 54.67 & 58.77 & 58.30 \\
\hline $\mathrm{TiO}_{2}$ & 0.00 & 0.03 & \multicolumn{2}{|c|}{0.02} & 0.00 & 0.00 & 0.00 & 0.01 & 0.00 & 0.02 & 0.04 & 0.06 & 0.05 & 0.07 & 0.03 & 0.07 & 0.03 \\
\hline $\mathrm{Al}_{2} \mathrm{O}_{3}$ & 0.01 & 0.00 & \multicolumn{2}{|c|}{0.05} & 0.05 & 0.00 & 0.02 & 0.02 & 0.00 & 1.31 & 1.97 & 1.43 & 1.27 & 1.90 & 0.94 & 0.07 & 1.05 \\
\hline $\mathrm{Cr}_{2} \mathrm{O}_{3}$ & 0.00 & 0.00 & \multicolumn{2}{|c|}{0.00} & 0.00 & 0.00 & 0.04 & 0.04 & 0.00 & 0.54 & 0.64 & 0.46 & 0.81 & 0.91 & 0.64 & 0.05 & 0.27 \\
\hline $\mathrm{FeO}$ & 8.85 & 8.52 & \multicolumn{2}{|c|}{19.82} & 17.26 & 6.50 & 3.78 & 15.08 & 20.76 & 5.88 & 5.55 & 6.73 & 1.87 & 1.62 & 1.95 & 1.84 & 1.53 \\
\hline $\mathrm{NiO}$ & 0.30 & 0.30 & \multicolumn{2}{|c|}{0.06} & 0.29 & 0.11 & 0.29 & 1.24 & 0.37 & 0.03 & 0.13 & 0.11 & 0.03 & 0.06 & 0.06 & 0.13 & 0.10 \\
\hline $\mathrm{MnO}$ & 0.15 & 0.13 & \multicolumn{2}{|c|}{0.66} & 0.56 & 0.03 & 0.01 & 0.01 & 0.00 & 0.14 & 0.15 & 0.16 & 0.08 & 0.10 & 0.11 & 0.10 & 0.05 \\
\hline $\mathrm{MgO}$ & 49.09 & 50.26 & \multicolumn{2}{|c|}{41.80} & 44.75 & 38.39 & 36.99 & 33.48 & 31.45 & 35.06 & 34.06 & 34.56 & 15.20 & 16.94 & 17.65 & 23.44 & 22.89 \\
\hline $\mathrm{CaO}$ & 0.05 & 0.03 & \multicolumn{2}{|c|}{0.01} & 0.00 & 0.00 & 0.00 & 0.03 & 0.00 & 0.43 & 0.44 & 0.40 & 23.46 & 23.96 & 25.22 & 12.97 & 13.23 \\
\hline $\mathrm{Na}_{2} \mathrm{O}$ & 0.02 & 0.03 & \multicolumn{2}{|c|}{0.00} & 0.03 & 0.00 & 0.02 & 0.00 & 0.00 & 0.02 & 0.00 & 0.00 & 0.16 & 0.04 & 0.25 & 0.05 & 0.25 \\
\hline $\mathrm{K}_{2} \mathrm{O}$ & 0.00 & 0.02 & \multicolumn{2}{|c|}{0.00} & 0.00 & 0.00 & 0.00 & 0.00 & 0.01 & 0.00 & 0.01 & 0.00 & 0.00 & 0.00 & 0.00 & 0.00 & 0.01 \\
\hline Total & 100.02 & 100.00 & \multicolumn{2}{|c|}{101.59} & 101.94 & 84.86 & 82.39 & 83.96 & 84.75 & 101.06 & 100.11 & 100.43 & 96.43 & 99.96 & 101.52 & 97.49 & 97.71 \\
\hline \multicolumn{18}{|c|}{ (b) } \\
\hline Mineral & \multicolumn{2}{|c|}{ Hornblende } & \multicolumn{5}{|c|}{ Chromite } & \multicolumn{2}{|c|}{ Magnetite } & \multicolumn{2}{|c|}{ Magnesite } & \multicolumn{2}{|c|}{ Fe-magnesite } & Dol & nite & & \\
\hline wt.\% & W12 & W12 & W4 & W12 & W6 & W6 & W7 & W16 & W5 & W6 & W15 & W7 & W7 & W5 & W7 & W4 & W7 \\
\hline $\mathrm{SiO}_{2}$ & 45.91 & 45.57 & 0.02 & 0.08 & 0.06 & 0.06 & 0.62 & 0.79 & 0.15 & 0.11 & 3.57 & 0.33 & 0.16 & 0.04 & 0.27 & 0.04 & 0.04 \\
\hline $\mathrm{TiO}_{2}$ & 0.29 & 0.27 & 0.03 & 0.11 & 0.39 & 0.35 & 0.99 & 0.02 & 0.03 & 0.04 & 0.02 & 0.00 & 0.00 & 0.00 & 0.03 & 0.01 & 0.00 \\
\hline $\mathrm{Al}_{2} \mathrm{O}_{3}$ & 10.01 & 10.24 & 27.34 & 16.03 & 0.82 & 1.31 & 0.71 & 0.08 & 0.00 & 0.01 & 2.06 & 0.03 & 0.00 & 0.01 & 0.03 & 0.00 & 0.01 \\
\hline $\mathrm{Cr}_{2} \mathrm{O}_{3}$ & 1.99 & 2.04 & 42.79 & 50.47 & 50.74 & 51.07 & 44.62 & 0.00 & 0.03 & 0.00 & 0.04 & 0.06 & 0.00 & 0.00 & 0.03 & 0.00 & 0.01 \\
\hline $\mathrm{FeO}$ & 2.55 & 2.69 & 17.05 & 22.20 & 43.93 & 42.47 & 46.25 & 91.58 & 93.35 & 0.26 & 6.26 & 26.37 & 23.18 & 2.56 & 1.98 & 0.12 & 0.20 \\
\hline $\mathrm{NiO}$ & 0.09 & 0.11 & 0.09 & 0.07 & 0.00 & 0.01 & 0.05 & 0.46 & 0.00 & 0.00 & 0.03 & 0.00 & 0.00 & 0.00 & 0.05 & 0.07 & 0.00 \\
\hline $\mathrm{MnO}$ & 0.01 & 0.01 & 0.15 & 0.17 & 0.58 & 0.54 & 0.57 & 0.56 & 1.11 & 0.22 & 0.07 & 0.81 & 0.70 & 2.39 & 0.31 & 0.00 & 0.00 \\
\hline $\mathrm{MgO}$ & 19.61 & 19.48 & 13.49 & 10.53 & 1.70 & 1.70 & 1.75 & 0.66 & 0.12 & 45.38 & 45.62 & 38.21 & 42.31 & 21.00 & 22.65 & 0.05 & 0.00 \\
\hline $\mathrm{CaO}$ & 13.41 & 13.44 & 0.00 & 0.01 & 0.02 & 0.03 & 0.03 & 0.00 & 0.04 & 2.03 & 0.80 & 0.05 & 0.01 & 26.53 & 31.83 & 61.96 & 57.94 \\
\hline $\mathrm{Na}_{2} \mathrm{O}$ & 3.19 & 3.09 & 0.03 & 0.02 & 0.00 & 0.00 & 0.00 & 0.04 & 0.00 & 0.00 & 0.01 & 0.00 & 0.01 & 0.02 & 0.00 & 0.00 & 0.00 \\
\hline $\mathrm{K}_{2} \mathrm{O}$ & 0.03 & 0.03 & 0.00 & 0.00 & 0.00 & 0.00 & 0.01 & 0.01 & 0.01 & 0.01 & 0.04 & 0.01 & 0.00 & 0.00 & 0.00 & 0.00 & 0.00 \\
\hline Total & 97.09 & 96.97 & 100.99 & 99.68 & 98.24 & 97.54 & 95.61 & 94.20 & 94.83 & 48.06 & 58.52 & 65.88 & 66.38 & 52.55 & 57.16 & 62.25 & 58.20 \\
\hline
\end{tabular}


Table 5. Representative analyses (EMPA) of the listvenite samples minerals.

\begin{tabular}{|c|c|c|c|c|c|c|c|c|c|c|c|c|c|c|c|c|c|c|}
\hline \multirow{2}{*}{$\begin{array}{l}\text { Mineral } \\
\text { wt. } \%\end{array}$} & \multicolumn{2}{|c|}{ Dolomite } & \multicolumn{2}{|c|}{ Plagioclase } & \multicolumn{2}{|c|}{ K-Feldspar } & \multicolumn{2}{|c|}{ Biotite } & \multicolumn{2}{|c|}{ Chlorite } & \multicolumn{2}{|c|}{ Amphibole } & \multicolumn{2}{|c|}{ Greenalite } & \multicolumn{2}{|c|}{ Chromite } & \multicolumn{2}{|c|}{ Fe-Chromite } \\
\hline & W1 & W17 & W1 & W1 & W1 & W1 & W1 & W1 & W2 & W2 & W1 & W1 & W17 & W17 & W2 & W17 & W2 & W2 \\
\hline $\mathrm{SiO}_{2}$ & 0.03 & 0.03 & 56.74 & 60.88 & 65.33 & 66.10 & 39.78 & 40.07 & 31.92 & 32.03 & 54.99 & 55.66 & 39.42 & 39.51 & 0.01 & 0.08 & 0.03 & 0.04 \\
\hline $\mathrm{TiO}_{2}$ & 0.00 & 0.03 & 0.00 & 0.00 & 0.00 & 0.04 & 1.50 & 1.34 & 0.02 & 0.08 & 0.23 & 0.40 & 0.09 & 0.03 & 0.22 & 0.22 & 0.29 & 0.25 \\
\hline $\mathrm{Al}_{2} \mathrm{O}_{3}$ & 0.05 & 0.00 & 26.38 & 24.42 & 17.98 & 18.32 & 14.41 & 14.23 & 17.71 & 16.89 & 3.66 & 3.50 & 0.40 & 0.09 & 14.70 & 14.80 & 9.91 & 10.86 \\
\hline $\mathrm{Cr}_{2} \mathrm{O}$ & 0.00 & 0.00 & 0.00 & 0.00 & 0.00 & 0.01 & 0.04 & 0.06 & 2.03 & 1.66 & 0.07 & 0.03 & 0.02 & 0.03 & 52.55 & 51.94 & 56.35 & 55.98 \\
\hline $\mathrm{FeO}$ & 0.03 & 0.13 & 0.04 & 0.00 & 0.07 & 0.00 & 7.78 & 7.77 & 2.11 & 2.07 & 5.87 & 5.94 & 46.69 & 48.21 & 22.91 & 22.26 & 26.75 & 25.64 \\
\hline $\mathrm{NiO}$ & 0.05 & 0.00 & 0.01 & 0.05 & 0.04 & 0.04 & 0.25 & 0.18 & 0.15 & 0.20 & 0.01 & 0.02 & 0.82 & 0.81 & 0.13 & 0.00 & 0.00 & 0.08 \\
\hline $\mathrm{MnO}$ & 0.01 & 0.00 & 0.00 & 0.02 & 0.05 & 0.00 & 0.09 & 0.11 & 0.00 & 0.01 & 0.21 & 0.22 & 0.02 & 0.05 & 0.17 & 0.19 & 0.15 & 0.11 \\
\hline $\mathrm{MgO}$ & 21.20 & 21.24 & 0.01 & 0.01 & 0.00 & 0.01 & 21.55 & 22.29 & 33.20 & 33.49 & 19.78 & 19.57 & 3.92 & 1.36 & 10.25 & 10.22 & 4.52 & 5.57 \\
\hline $\mathrm{CaO}$ & 30.81 & 32.54 & 9.46 & 6.57 & 0.03 & 0.03 & 0.19 & 0.14 & 0.00 & 0.03 & 12.01 & 12.54 & 0.19 & 0.21 & 0.02 & 0.02 & 0.00 & 0.00 \\
\hline $\mathrm{Na}_{2} \mathrm{O}$ & 0.02 & 0.02 & 6.22 & 7.93 & 0.94 & 0.72 & 0.05 & 0.02 & 0.00 & 0.00 & 0.45 & 0.34 & 0.20 & 0.05 & 0.00 & 0.01 & 0.02 & 0.05 \\
\hline $\mathrm{K}_{2} \mathrm{O}$ & 0.00 & 0.01 & 0.22 & 0.23 & 15.14 & 15.42 & 5.68 & 5.52 & 0.02 & 0.01 & 0.16 & 0.13 & 0.22 & 0.00 & 0.00 & 0.01 & 0.00 & 0.00 \\
\hline Total & 52.21 & 54.00 & 99.07 & 100.11 & 99.58 & 100.68 & 91.32 & 91.73 & 87.16 & 86.46 & 97.44 & 98.36 & 91.98 & 90.34 & 100.96 & 99.75 & 98.02 & 98.59 \\
\hline
\end{tabular}


Plagioclase, K-feldspar and biotite are also abundant within $\mathrm{Al}_{2} \mathrm{O}_{3}$-rich listvenites. Plagioclase composition varies from An30 to An47. K-feldspar (Or92.5) was found only in one sample. Mica (biotite) is characterized by $\mathrm{Al}_{2} \mathrm{O}_{3}$ between 14.23 and $14.52 \mathrm{wt} . \%$, $\mathrm{FeO}$ between 7.69 and $7.78 \mathrm{wt} . \%$ and $\mathrm{MgO}$ between 21.55 and $22.29 \mathrm{wt} . \%$. $\mathrm{K}_{2} \mathrm{O}$ content is lower than typical biotite, ranging between 5.52 and $5.68 \mathrm{wt} . \%$, and it is enriched in $\mathrm{TiO}_{2}$, with values ranging between 1.23 and $1.50 \mathrm{wt} . \%$.

Other accessory phases include chlorite, amphibole, serpentine and chromite. Chlorite crystals are $\mathrm{Cr}$-chlinochlore, characterized by $\mathrm{MgO}$ between 33.20 and $33.49 \%$, $\mathrm{FeO}$ between 2.07 and $2.11 \%$ and $\mathrm{Cr}_{2} \mathrm{O}_{3}$ between 1.66 and $2.03 \%$. Amphibole is characterized by an homogeneous composition in all the listvenitic samples, with $\mathrm{MgO}$ ranging from 19.57 to $19.97 \mathrm{wt} . \%$, $\mathrm{FeO}$ between 5.65 and $5.94 \mathrm{wt} . \%$ and $\mathrm{CaO}$ between 12.01 and $12.54 \mathrm{wt} . \%$, and hence it can be classified as actinolite. In sample W17, the serpentine-group mineral greenalite was found to be characterized by the $\mathrm{FeO}$ content between 46.69 and $48.21 \mathrm{wt} . \%$ and a low $\mathrm{MgO}$ content (1.36-3.92 wt.\%). Spinels are mainly chromite and ferrian chromite rims and show an homogeneous composition in all listvenites. In chromites, $\mathrm{MgO}$ varies between 8.05 and 10.25 wt. $\%, \mathrm{Cr}_{2} \mathrm{O}_{3}$ between 47.14 and $53.90 \mathrm{wt} . \%$, FeO between 22.03 and 23.88 wt.\%, and $\mathrm{Al}_{2} \mathrm{O}_{3}$ between 14.59 and 18.28 wt. $\%$. Ferrian chromite has lower $\mathrm{MgO}$ and $\mathrm{Al}_{2} \mathrm{O}_{3}$ contents than chromite, with values ranging between 4.52 and $5.57 \mathrm{wt.} \%$ and 9.91 and $12.06 \mathrm{wt} . \%$, respectively, and higher $\mathrm{FeO}$ and $\mathrm{Cr}_{2} \mathrm{O}_{3}$ contents, ranging between 55.22 and $56.35 \mathrm{wt} . \%$ and between 25.41 and $26.75 \mathrm{wt} . \%$, respectively.

\subsubsection{Serpentinite with Clay Minerals}

Sample W3 has a peculiar mineralogical composition (Table 6), being a serpentinite with abundant plagioclase and clay minerals. The dominant mineral phases are serpentine, plagioclase, chlorite and amphibole, whereas clay minerals are mainly vermiculites.

Table 6. Representative analyses (EMPA) of the sample W3 minerals.

\begin{tabular}{cccccccccc}
\hline Mineral & \multicolumn{2}{c}{ Plagioclase } & \multicolumn{3}{c}{ Chlorite } & & Amphibole & \multicolumn{2}{c}{$\begin{array}{c}\text { Mg-Al } \\
\text { Hydroxides }\end{array}$} \\
\hline $\mathrm{SiO}_{2}$ & 57.66 & 57.44 & 39.57 & 40.28 & 34.95 & 55.85 & 56.24 & 1.74 & 1.24 \\
$\mathrm{TiO}_{2}$ & 0.00 & 0.00 & 0.82 & 0.76 & 0.01 & 0.05 & 0.05 & 0.00 & 0.00 \\
$\mathrm{Al}_{2} \mathrm{O}_{3}$ & 26.25 & 25.98 & 13.97 & 14.50 & 23.02 & 3.21 & 3.02 & 28.16 & 29.09 \\
$\mathrm{Cr}_{2} \mathrm{O}_{3}$ & 0.00 & 0.00 & 0.20 & 0.18 & 0.04 & 0.05 & 0.11 & 0.00 & 0.00 \\
$\mathrm{FeO}$ & 0.05 & 0.05 & 5.68 & 5.13 & 1.31 & 4.59 & 5.24 & 1.04 & 0.96 \\
$\mathrm{NiO}$ & 0.06 & 0.01 & 0.20 & 0.11 & 0.03 & 0.08 & 0.09 & 0.03 & 0.00 \\
$\mathrm{MnO}$ & 0.05 & 0.06 & 0.11 & 0.11 & 0.00 & 0.16 & 0.20 & 0.01 & 0.00 \\
$\mathrm{MgO}$ & 0.01 & 0.00 & 23.03 & 23.20 & 21.23 & 21.02 & 21.30 & 34.80 & 36.01 \\
$\mathrm{CaO}$ & 9.07 & 9.06 & 0.10 & 0.04 & 0.26 & 12.03 & 11.36 & 0.01 & 0.00 \\
$\mathrm{Na}{ }_{2} \mathrm{O}$ & 6.49 & 6.50 & 0.00 & 0.00 & 0.01 & 0.49 & 0.48 & 0.00 & 0.00 \\
$\mathrm{~K}_{2} \mathrm{O}$ & 0.17 & 0.19 & 0.24 & 0.05 & 0.05 & 0.05 & 0.06 & 0.01 & 0.00 \\
\hline $\mathrm{Total}$ & 99.80 & 99.29 & 83.91 & 84.36 & 80.91 & 97.58 & 98.15 & 65.80 & 67.31 \\
\hline
\end{tabular}

Plagioclase (An43.5) has a composition comparable to the one in listvenites. Amphibole is characterized by an homogeneous composition, comparable to those of actinolites in listvenites. $\mathrm{MgO}$ contents range between 20.56 and $21.30 \mathrm{wt}$ \%, FeO between 4.59 and $5.24 \mathrm{wt} . \%$, CaO between 11.36 and 12.03 and $\mathrm{Al}_{2} \mathrm{O}_{3}$ between 3.02 and $3.31 \mathrm{wt} . \%$.

\section{Discussion}

Based on the mineralogical assemblage, bulk rock geochemical data and EMPA analyses, we can trace the succession of events that affected the Rachoni massif peridotites. The peculiar mineral assemblage is indicative of several metasomatic events affecting the area. After a first stage of serpentinization, $\mathrm{CO}_{2}$-rich fluids percolated through the rocks replacing the mineralogical assemblage 
with a suite of new formed minerals. Locally, the carbonation of ultramafic rocks led to the formation of listvenites (Figure 6A), with the precipitation of quartz and K-mica along with carbonates.

\subsection{Genesis of the Protolith}

The composition of unaltered chromites in the Rachoni peridotites is variable, with $\mathrm{Cr}_{2} \mathrm{O}_{3}$ contents between 34.53 and 53.90 wt. $\%, \mathrm{Al}_{2} \mathrm{O}_{3}$ between 14.68 and 34.63 wt. $\%$ and $\mathrm{TiO}_{2}$ up to 0.33 wt.\%. Their composition is comparable to the one of spinels plotting within the ophiolite compositional field (Figure 9A,B). Rachoni peridotite spinels are characterized by relatively high $\mathrm{XCr}$ and low $\mathrm{XMg}$, and plot in the forearc peridotite genetic field (Figure 9C). Such character is in agreement with previous works on chromitite spinels from Gerakini [23] and Vavdos [24] ophiolites, that are within the podiform chromitites field plot in the area of low XMg.

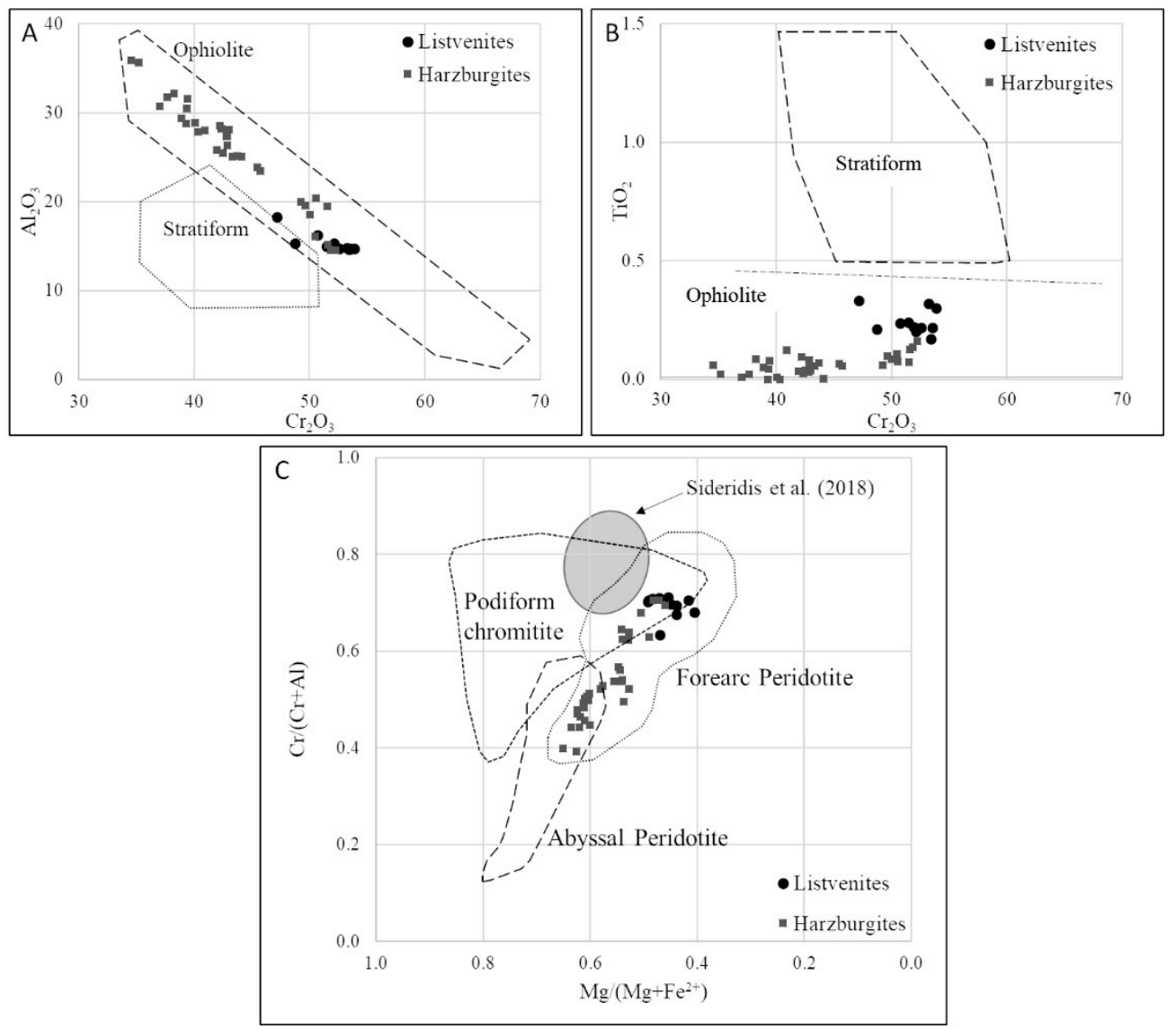

Figure 9. $\mathrm{Cr}_{2} \mathrm{O}_{3}$ vs. $\mathrm{Al}_{2} \mathrm{O}_{3}$ (A) and $\mathrm{TiO}_{2}$ (B) of the Rachoni peridotite chromites; (C) $\mathrm{Mg} /\left(\mathrm{Mg}+\mathrm{Fe}^{2+}\right)$ vs. $\mathrm{Cr} /(\mathrm{Cr}+\mathrm{Al})$ of Rachoni peridotites chromite compared to chromitite chromites from the same area. Compositional fields for abyssal peridotites [25-27], forearc peridotites [26] and podiform chromitites $[23,24,28]$ are shown for comparison.

\subsection{Fe-Chromitization}

Alteration into ferrian chromite in the Rachoni peridotites is unevenly distributed and limited to a few samples. The general ferrian chromitization trend shows a depletion in $\mathrm{Al}$, with a relative enrichment in $\mathrm{Cr}$ and $\mathrm{Fe}^{3+}$, coupled with an important $\mathrm{Mg}$ depletion (Figure 10). Ferrian chromitization is generally confined to chromite rims, and only in rare cases it affects the whole chromite grain. Cr-chlorite development, which is generally associated to ferrian chromite alteration [29], is absent in our samples. The uneven distribution of ferrian chromitization, not correlated to that of listvenites and not as widespread as serpentinization, suggests that it can be related to the limited circulation of low-T fluids. 


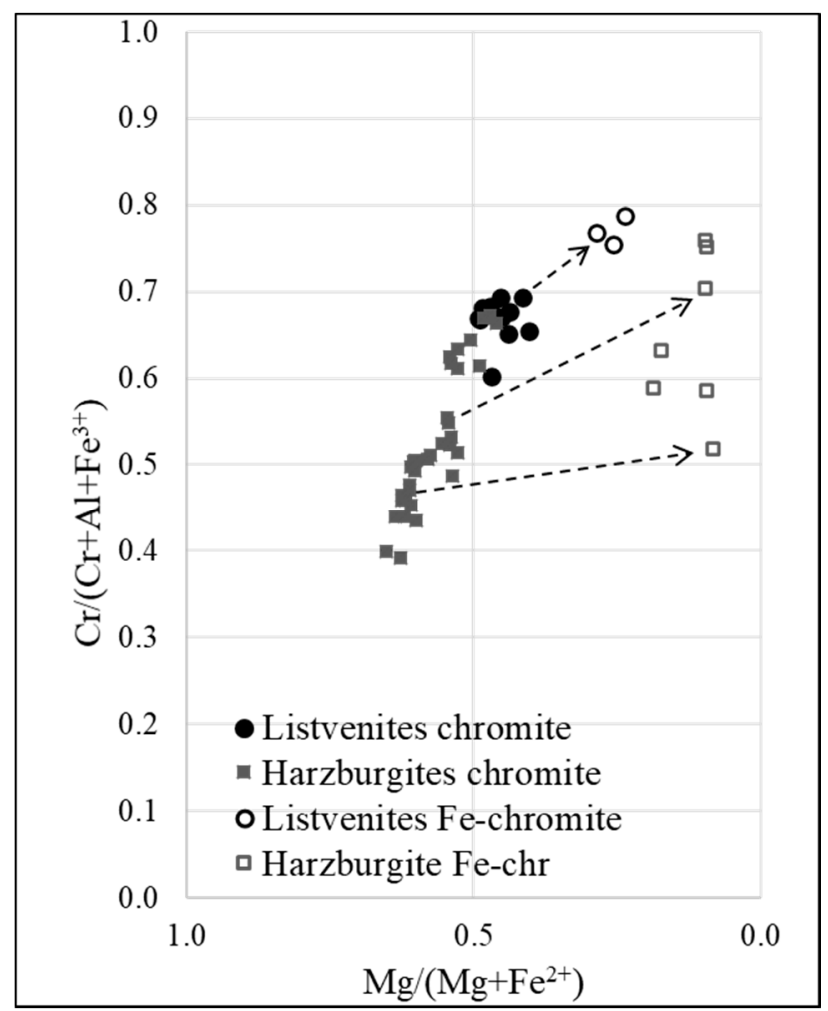

Figure 10. $\mathrm{Mg} /\left(\mathrm{Mg}+\mathrm{Fe}^{2+}\right)$ vs. $\mathrm{Cr} /\left(\mathrm{Cr}+\mathrm{Al}+\mathrm{Fe}^{3+}\right)$ of chromites and ferrian chromites from listvenites and peridotites; the arrows indicate ferrian chromitization evolution.

\subsection{Serpentinization}

Textural and petrographic observations indicate that serpentinization is the first alteration stage of the peridotites. The starting hydration reaction occurring at the first stages of serpentinization, at temperatures of $\sim 400{ }^{\circ} \mathrm{C}$, is the conversion of olivine to serpentine following reaction (1) [30]:

$$
\underset{3 \mathrm{Mg}_{2} \mathrm{SiO}_{4}}{3}+\underset{\text { Olivine fluid }}{2 \mathrm{SiO}_{2}}+4 \mathrm{H}_{2} \mathrm{O}=\underset{\text { serpentine }}{2 \mathrm{Mg}_{3} \mathrm{Si}_{2} \mathrm{O}_{5}(\mathrm{OH})_{4}}
$$

According to the XRD data and petrographic observations, we can infer that peridotite samples from the Rachoni mine underwent serpentinization at high silica activity, which prevented the formation of brucite, which was not detected.

Orthopyroxene, as well as olivine, is also involved in hydration reactions. The reaction of orthopyroxene with silica-rich and water-rich fluids results in the precipitation of serpentine and talc, following reaction (2) [30]:

$$
\begin{array}{ccc}
6 \mathrm{MgSiO}_{3} & +\underset{\mathrm{H}}{\mathrm{H}_{2} \mathrm{O}}=\underset{\mathrm{Opx}}{\mathrm{Mg}_{3} \mathrm{Si}_{2} \mathrm{O}_{5}(\mathrm{OH})_{4}}+\underset{\mathrm{Mg}_{3} \mathrm{Si}_{4} \mathrm{O}_{10}(\mathrm{OH})_{2}}{\text { fluid }} \text { serpentine } & \text { talc }
\end{array}
$$

The development of Fe-rich hydrated phases during serpentinization, observed in some samples, suggests that during initial serpentinization, iron partitions from olivine and pyroxene into lizardite and brucite, as observed also in other harzburgites [31], according to reaction (3):

$$
\begin{gathered}
2 \mathrm{Fe}_{2} \mathrm{SiO}_{4} \\
\text { Olivine fluid }
\end{gathered}
$$

With proceeding serpentinization, partial iron release from early formed Fe-hydrated phases led to the formation of magnetite (Figure 5D) $[30,32,33]$. 


\subsection{Fe Inclusions in Olivine}

During the examination with SEM of sample W5, a very rare phenomenon was observed: olivine grains had inclusions (Figure 11), which the EDS and WDS analyses confirmed consisted of native Fe.

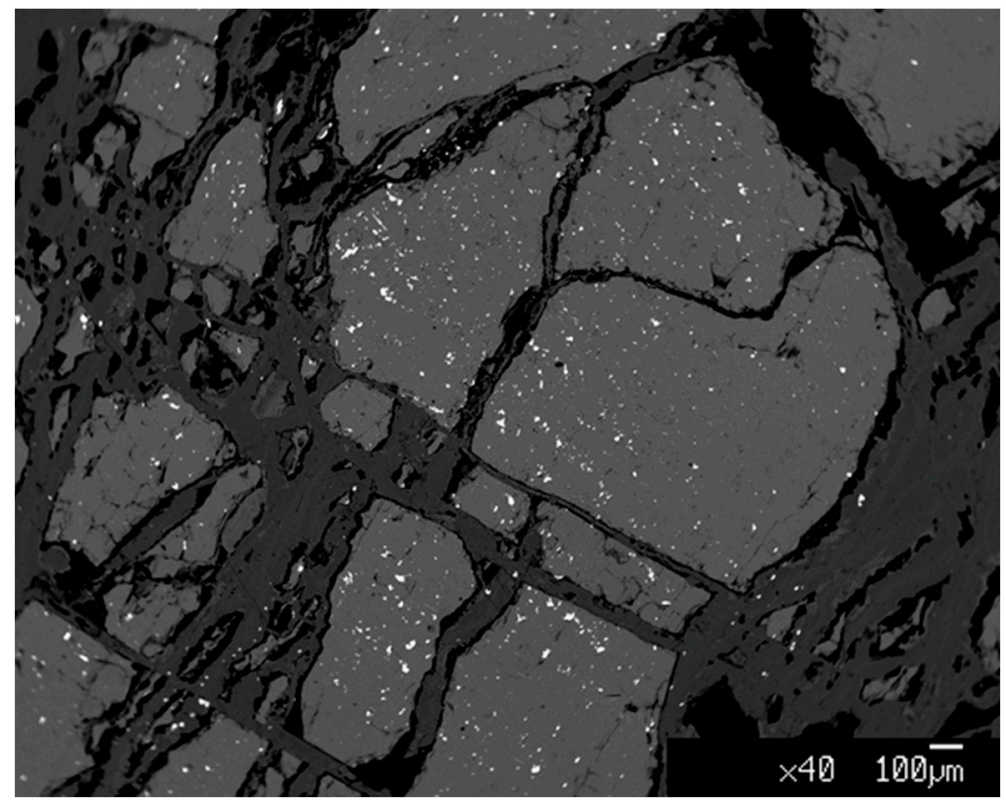

Figure 11. Back scattered electron (BSE) image from sample W5 showing Fe inclusions (white) in olivine relics into serpentine matrix.

The study of the mineral chemistry of olivines from sample W5 (Table 7) did not reveal any notable differences between the olivine grains hosting Fe inclusions and those not. All olivines were found with a high Fo content; Fo shows a constant value of $0.90\left(\mathrm{Fo}_{0.90} \mathrm{Fa}_{0.10}\right)$. The $\mathrm{NiO}$ content of olivines is rather low $(<0.44 \mathrm{wt} . \%)$ and no Ni alloys were found during the microscopic study of the sample.

Table 7. Mineral chemistry of olivine grains from sample W5. Analyses No. 22-26 are from the olivine grains with Fe inclusions, whereas analyses No. 31-35 are from olivine grains without inclusions ( $-=$ below detection limit).

\begin{tabular}{ccccccccccc}
\hline Analysis & $\mathbf{2 2}$ & $\mathbf{2 3}$ & $\mathbf{2 4}$ & $\mathbf{2 5}$ & $\mathbf{2 6}$ & $\mathbf{3 1}$ & $\mathbf{3 2}$ & $\mathbf{3 3}$ & $\mathbf{3 4}$ & $\mathbf{3 5}$ \\
\hline $\mathrm{SiO}_{2}$ & 41.59 & 41.51 & 41.75 & 41.30 & 41.16 & 41.65 & 41.75 & 41.31 & 41.31 & 41.74 \\
$\mathrm{TiO}_{2}$ & 0.01 & 0.04 & 0.05 & 0.01 & 0.05 & - & - & - & 0.01 & - \\
$\mathrm{Al}_{2} \mathrm{O}_{3}$ & - & - & - & 0.04 & - & - & - & - & 0.02 & 0.02 \\
$\mathrm{Cr}_{2} \mathrm{O}_{3}$ & - & 0.01 & - & - & - & - & 0.04 & - & 0.04 & 0.03 \\
$\mathrm{FeO}$ & 9.04 & 8.98 & 8.94 & 9.03 & 8.96 & 9.17 & 8.97 & 9.01 & 8.89 & 8.94 \\
$\mathrm{MnO}$ & 0.12 & 0.15 & 0.15 & 0.15 & 0.10 & 0.08 & 0.13 & 0.11 & 0.10 & 0.14 \\
$\mathrm{MgO}$ & 49.66 & 50.00 & 49.58 & 49.60 & 49.61 & 49.73 & 49.74 & 49.84 & 50.06 & 49.38 \\
$\mathrm{NiO}$ & 0.36 & 0.39 & 0.41 & 0.44 & 0.32 & 0.36 & 0.29 & 0.36 & 0.38 & 0.39 \\
$\mathrm{CaO}$ & 0.03 & 0.03 & 0.02 & 0.03 & 0.03 & 0.03 & 0.02 & 0.01 & 0.01 & 0.01 \\
\hline $\mathrm{Total}$ & 100.82 & 101.11 & 100.90 & 100.60 & 100.23 & 101.03 & 100.93 & 100.65 & 100.83 & 100.65 \\
\hline Fo & 0.90 & 0.90 & 0.90 & 0.90 & 0.90 & 0.90 & 0.90 & 0.90 & 0.90 & 0.90 \\
\hline
\end{tabular}

Native Fe has been found in ophiolitic rocks such as serpentinized dunites, peridotites, serpentinites and chromitites [34]. In most cases, native Fe presence is associated with serpentinization, e.g., [35-39]. Sakai and Kuroda [39] suggested that during serpentinization, Fe is released from olivine forming magnetite; hydrogen is also produced during serpentinization as indicated by the reaction [35]:

$$
6 \mathrm{Mg}_{1.5} \mathrm{Fe}_{0.5} \mathrm{SiO}_{4}+7 \mathrm{H}_{2} \mathrm{O}=3 \mathrm{Mg}_{3} \mathrm{Si}_{2} \mathrm{O}_{5}(\mathrm{OH})_{4}+\mathrm{Fe}_{3} \mathrm{O}_{4}+\mathrm{H}_{2}
$$


Hydrogen could theoretically reduce magnetite to $\mathrm{Fe}$, but it is uncertain if the low temperatures during serpentinization would allow magnetite to be affected. Furthermore, native Fe in the aforementioned cases [35-39] is found in serpentine veins; in our sample, Fe was found as inclusions in olivine.

In order to explain the Fe inclusions in olivine, we propose that they could have segregated from magmas prior to or along with the crystallization of olivine. The segregation of these inclusions, although a minor amount, could result in Fe depletion in the evolved magmas [40]. Nevertheless, subsolidus equilibration between olivine and chromite $\left(\mathrm{Mg}^{2+}\right.$ diffusion from chromite to olivine and $\mathrm{Fe}^{2+}$ from olivine to chromite at a subsolidus temperature) is the most probable factor controlling the olivine chemical composition and its high Fo content.

\subsection{Carbonation}

Carbonation of Rachoni harzburgites is characterized by the precipitation of carbonate phases, mainly magnesite, calcite and dolomite. The paragenetic sequence of carbonated ultramafic rocks has been studied by several authors, who suggest that a serpentinite can be transformed into a talc-rich rock and/or into a quartz-carbonate-bearing rock by interaction with $\mathrm{CO}_{2}$-rich hydrothermal fluids [41,42], derived from seawater and the atmosphere [42]. When serpentinized peridotites come into contact with such fluids, they are partially or completely replaced by talc-magnesite and/or quartz-magnesite rocks [43-46]. Textural observations reveal that magnesite in the Rachoni harzburgites preferentially replaces serpentine and pyroxenes, as observed in other carbonated serpentinites [43]. The reaction suggested for this process involves the interaction of lizardite/chrysotile with $\mathrm{CO}_{2}$-rich fluids to form antigorite and magnesite [31,47].

Minor carbonate phases found in the Rachoni peridotites are Fe-rich magnesite, dolomite and calcite. Fe-rich magnesite, found only locally, results from the replacement of olivine instead of serpentine [42], whereas dolomite and calcite are the result of the alteration of clinopyroxene crystals [31] by the same $\mathrm{CO}_{2}$-rich fluids.

\subsection{Plagioclase Formation}

Sample W3 shows a peculiar mineralogical association that could be related to a further alteration stage before listvenitization. The XRPD and mineral chemistry data reveal the presence of serpentine, magnesite, amphibole, chlorite and talc, very similar to the association in partially serpentinized harzburgites, but also of plagioclase. While the major element content of sample W3 is similar to the one in harzburgites, the REE content is higher, more similar to the content found in the listvenite of the Rachoni complex. This enrichment is due to Na-rich percolating fluids, also responsible for the precipitation of plagioclase.

\subsection{Listvenitization}

Areas of strong listvenitization can be found within the ultramafic rocks of Rachoni mine, crosscut by later magnesite veins (Figure 6A). The main effect is carbonation in the presence of K-bearing fluids, with the replacement of primary minerals by an assemblage of $\mathrm{Mg}-\mathrm{Fe}(-\mathrm{Ca})$ carbonates, quartz and chromian muscovite or sericite [48]. There is a mineralogical distinction between talc-carbonate rocks and listvenites, which is the presence of potassium in listvenites [49]. However, listvenitization is also commonly used to describe carbonated ultramafic rocks, and the term has already been used to describe similar lithologies in the ophiolite mélange of Iti mountain, in central continental Greece [49].

Quartz is present in all listvenite samples but is the most abundant in sample W17. Carbonation reactions and the influx of $\mathrm{CO}_{2}$ can lead to quartz oversaturation and its co-precipitation with magnesite [31]. Quartz and magnesite can also result from the reaction of talc and $\mathrm{CO}_{2}$-rich fluids. The absence of talc in the Rachoni listvenites could indicate its complete consumption in this second reaction. In contrast to "classic" listvenite, K-bearing minerals in Rachoni are not Cr-muscovite or sericite, but biotite and K-feldspar. In Ugo mine (W17), no K-bearing phase was found. 
REE patterns (Figure 8) in listvenitic samples are generally flat but show an enrichment in LREE with respect to HREE. The two listvenites from Rachoni mine are highly enriched in LREE, with a content similar to plagioclase-enriched sample W3, whereas sample W17 (Ugo mine), although containing the highest $\mathrm{SiO}_{2}$ content, is less enriched in rare earth elements. Rachoni listvenites and plagioclase-enriched serpentinite, moreover, present a small to accentuated Eu-negative anomaly, which is absent in listvenite W17.

The REE enrichment in samples W1 to W3 could have been inherited from the same circulating fluids, percolating through the rock along fractures and pores and enriching the host lithology in incompatible elements. The enrichment in LREE also suggests a strong contribution of carbonates, since $\mathrm{CO}_{3}{ }^{-2}$ ions fractionate LREE relative to HREE [50].

\section{Conclusions}

According to the results of our study, the Gerakini ophiolite shows signs of having undergone various stages of metamorphism/matasomatism. The Fe inclusions observed in some olivine grains must have segregated from magmas prior to or along with the crystallization of olivine and the segregation of these inclusions, which could result in Fe depletion in the evolved magmas.

After the initial serpentinization event (i.e., ocean floor metamorphism), $\mathrm{CO}_{2}$-rich fluids circulated into these rocks resulting in the further alteration of these rocks and the formation of new mineral phases with magnesite being the most important one due its economic interest. Furthermore, locally, the rocks were affected by silicification, carbonation and clay alterations, resulting in the formation of listvenites and clay mineral phases in the serpentinites.

The serpentinization of the rocks resulted in the formation of serpentine, whereas brucite was not found in these samples; the latter along with the presence of olivine relics, implies that serpentinization stopped before the final stage of brucite formation.

The post-serpentinization circulation of $\mathrm{CO}_{2}$-rich fluids in these rocks resulted in the formation of antigorite and magnesite from the carbonation of lizardite and/or chrysotile and the formation of Fe-magnesite from the carbonation of olivine, whereas dolomite and calcite were formed by the effect of the same fluids into clinopyroxenes.

During carbonation, the local development of the listvenites, resulting in the depletion of $\mathrm{Mg}, \mathrm{Mn}$, HREE, and Pd, and to the enrichment in $\mathrm{Si}, \mathrm{Ca}, \mathrm{Ba}, \mathrm{Zr}, \mathrm{LREE}$, in the listvenites; Ti, $\mathrm{Cr}, \mathrm{Ni}$ and Fe have been conserved. Two different fluids were involved in the evolution of these rocks as inferred by the differences in the REE contents of listvenites from two different localities of the Gerakini ophiolites.

Author Contributions: Conceptualization, E.T, M.B. and G.G.; methodology, E.T., A.K. and A.Z.; validation, E.T., A.K., P.M. and L.C.; formal analysis, M.B., E.T. and G.G.; resources, E.T., A.K. and A.Z.; data curation, E.T., A.K., N.S., K.S. and A.Z.; writing-original draft preparation, E.T., M.B. and G.G.; writing-review and editing, E.T., M.B. and G.G.; supervision, E.T. and A.Z; project administration, E.T. and A.Z. All authors have read and agreed to the published version of the manuscript.

Funding: This research has been co-financed by the European Union and Greek national funds through the Operational Program Competitiveness, Entrepreneurship and Innovation, under the call RESEARCH - CREATE INNOVATE (project code: T1EDK-03543). The work was partially supported for the Italian co-authors by the Italian Ministry of Education (MIUR) through the projects "PRIN2017 - Mineral reactivity, a key to understand large-scale processes" (2017L83S77) and "Dipartimenti di Eccellenza 2018-2022".

Acknowledgments: Specific thanks are due to X. Dambou (Chemist, MSc, Dept. of Chem. Eng., Aristotle University) for the production and elaboration of XRD measurements and to A. Risplendente (Technician, Earth Sciences Department of the University of Milan) for the EMPA.

Conflicts of Interest: The authors declare no conflict of interest.

\section{References}

1. Pagona, E.; Tzamos, E.; Zouboulis, A.; Mitrakas, M. Characterization and evaluation of by-products from magnesite mines of Gerakini Chalkidiki. In Proceedings of the 12th Panhellenic Scientific Conference in Chemical Engineering, Athens, Greece, 29-31 May 2019; p. 5. 
2. Pagona, E.; Tzamos, E.; Simeonidis, K.; Zouboulis, A.; Mitrakas, M. Characterization and evaluation of magnesite ore mining procedures by-products from Gerakini mines, Chalkidiki, Greece. In Proceedings of the 7th International Conference on Sustainable Solid Waste Management, Herakleion, Greece, 26-29 June 2019; p. 8.

3. Pagona, E.; Tzamos, E.; Grieco, G.; Zouboulis, A.; Mitrakas, M. Characterization and evaluation of magnesite ore mining by-products of Gerakini mines (Chalkidiki, N. Greece). Sci. Total Environ. 2020, 732, 139279. [CrossRef] [PubMed]

4. Papadopoulos, A.; Tzamos, E.; Pagona, E.; Grieco, G.; Kasinos, A.; Tziritis, E.; Mitrakas, M.; Zouboulis, A. Mineralogical and geochemical investigation of the alteration of ultramafic magnesite-hosting rocks from Gerakini (Chalkidiki, Northern Greece). In Proceedings of the 15th International Congress of the Geological Society of Greece, Bulletin Geological Society Greece, Athens, Greece, 22-24 May 2019.

5. Tzamos, E.; Grieco, G.; Pagona, E.; Papadopoulos, A.; Bussolesi, M.; Mitrakas, M.; Zouboulis, A. First report of Fe inclusions in olivine: Olivine from the magnesite-hosting ultramafic rocks of Gerakini, Chalkidiki, Northern Greece. In Proceedings of the 15th International Congress of the Geological Society of Greece, Bulletin Geological Society Greece, Athens, Greece, 22-24 May 2019.

6. Tzamos, E.; Simeonidis, K.; Pagona, E.; Ntampou, X.; Zouboulis, A.; Mitrakas, M. Laboratory evaluation of ultramafic rocks exploitation towards the production of added-value products. In Proceedings of the 11th International Congress on Instrumental Methods of Analysis: Modern Trends and Applications, Ioannina, Greece, 22-25 September 2019; p. 159.

7. McCollom, T.M.; Bach, W. Thermodynamic constraints on hydrogen generation during serpentinization of ultramafic rocks. Geochim. Cosmochim. Acta 2009, 73, 856-875. [CrossRef]

8. Abu-Jaber, N.S.; Kimberley, M.M. Origin of ultramafic-hosted vein magnesite deposits. Ore Geol. Rev. 1992, 7, 155-191. [CrossRef]

9. Pohl, W. Genesis of magnesite deposits-Models and trends. Geol. Rundsch. 1990, 79, 291-299. [CrossRef]

10. Zachmann, D.W.; Johannes, W. Cryptocrystalline Magnesite. Magnesite Monogr. Ser. Miner. Depos. 1989, $28,15-28$.

11. Jedrysek, M.O.; Halas, S. The origin of magnesite deposits from the Polish Foresudetic Block ophiolites: Preliminary $\delta 13 \mathrm{C}$ and $\delta 18 \mathrm{O}$ investigations. Terra Nova 1990, 2, 154-159. [CrossRef]

12. Gartzos, E. Comparative stable isotope study of the magnesite deposits of Greece. Bull. Geol. Soc. Greece 2004, 36, 196-203. [CrossRef]

13. Ilich, M.; Toshovich, R. Geology and Origin the Golesh Vein Magnesite Deposit: A Brief Survey. 2005. Available online: http://www.geologicacarpathica.com/data/files/files/special\%20issue/I/Ilich_Toshovich.pdf (accessed on 10 July 2020).

14. Kelemen, P.B.; Matter, J. In situ carbonation of peridotite for $\mathrm{CO}_{2}$ storage. Proc. Natl. Acad. Sci. USA 2008, 105, 17295-17300. [CrossRef]

15. Kelemen, P.B.; Matter, J.; Streit, E.E.; Rudge, J.F.; Curry, W.B.; Blusztajn, J. Rates and mechanisms of mineral carbonation in peridotite: Natural processes and recipes for enhanced, in situ $\mathrm{CO} 2$ capture and storage. Annu. Rev. Earth Planet. Sci. 2011, 39, 545-576. [CrossRef]

16. Lesko, I. Über die Bildung von Magnesitlagerstätten. Miner. Depos. 1972, 7. [CrossRef]

17. Edelstein, I.; Melnik, A.; Pilipenko, A.A. On geochemistry of process of eathering of the rock-forming minerals of ultramafic rocks (according to experimental data). Geochemia 1982, 2, 263-570.

18. Rose, G. Mineralogisch-Geognostische Reise Nach dem Ural, dem Altai und dem Kaspischen Meere, Volume 1: Reise nach dem Nordlichen Ural und dem Altai; Forgotten Books: Berlin, Germany, 1837.

19. Tsirampides, A.; Filippidis, A. Greek Mineral Resources of Greece: Reserves and Value; Aristotle University of Thessaloniki: Thessaloniki, Greece, 2013; 46p.

20. Kauffmann, G.; Kockel, F.; Mollat, H. Notes on the stratigraphic and paleogeographic position of the Svoula Formation in the innermost zone of the Hellenides (northern Greece). Bull. Société Géologique Fr. 1976, S7-XVIII, 225-230. [CrossRef]

21. Meinhold, G.; Kostopoulos, D.K. The Circum-Rhodope Belt, northern Greece: Age, provenance, and tectonic setting. Tectonophysics 2013, 595-596, 55-68. [CrossRef]

22. McDonough, W.F.; Sun, S.-S. The composition of the Earth. Chem. Geol. 1995, 120, 223-253. [CrossRef] 
23. Sideridis, A.; Zaccarini, F.; Grammatikopoulos, T.; Tsitsanis, P.; Tsikouras, B.; Pushkarev, E.; Garuti, G.; Hatzipanagiotou, K. First occurrence of Ni-phosphides in chromitites from the ophiolite complexes of Alapaevsk, Russia and Gerakini-Ormylia, Greece. Ofioliti 2018, 43, 75-84. [CrossRef]

24. Grieco, G.; Pedrotti, M.; Moroni, M. Metamorphic redistribution of Cr within chromitites and its influence on chromite ore enrichment. Miner. Eng. 2011, 24, 102-107. [CrossRef]

25. Arai, S. Characterization of spinel peridotites by olivine-spinel compositional relationships: Review and interpretation. Chem. Geol. 1994, 113, 191-204. [CrossRef]

26. Arai, S.; Okamura, H.; Kadoshima, K.; Tanaka, C.; Suzuki, K.; Ishimaru, S. Chemical characteristics of chromian spinel in plutonic rocks: Implications for deep magma processes and discrimination of tectonic setting. Isl. Arc 2011, 20, 125-137. [CrossRef]

27. Dick, H.J.B.; Bullen, T. Chromian spinel as a petrogenetic indicator in abyssal and alpine-type peridotites and spatially associated lavas. Contrib. Mineral. Petrol. 1984, 86, 54-76. [CrossRef]

28. Miura, M.; Arai, S. Platinum-group element and mineral characteristics of sub-arc chromitite xenoliths from the Takashima alkali basalt, southwest Japan. Can. Mineral. 2014, 52, 899-916. [CrossRef]

29. Merlini, A.; Grieco, G.; Diella, V. Ferritchromite and chromian-chlorite formation in mélange-hosted Kalkan chromitite (Southern Urals, Russia). Am. Mineral. 2009, 94, 1459-1467. [CrossRef]

30. Frost, R.B.; Beard, J.S. On silica activity and serpentinization. J. Petrol. 2007, 48, 1351-1368. [CrossRef]

31. Boskabadi, A.; Pitcairn, I.K.; Leybourne, M.I.; Teagle, D.A.H.; Cooper, M.J.; Hadizadeh, H.; Nasiri Bezenjani, R.; Monazzami Bagherzadeh, R. Carbonation of ophiolitic ultramafic rocks: Listvenite formation in the Late Cretaceous ophiolites of Eastern Iran. Lithos 2020, 352-353, 105307. [CrossRef]

32. Frost, B.R.; Evans, K.A.; Swapp, S.M.; Beard, J.S.; Mothersole, F.E. The process of serpentinization in dunite from New Caledonia. Lithos 2013, 178, 24-39. [CrossRef]

33. Klein, F.; Bach, W.; Jöns, N.; McCollom, T.; Moskowitz, B.; Berquó, T. Iron partitioning and hydrogen generation during serpentinization of abyssal peridotites from $15^{\circ} \mathrm{N}$ on the Mid-Atlantic Ridge. Geochim. Cosmochim. Acta 2009, 73, 6868-6893. [CrossRef]

34. Kanellopoulos, C.; Valsami-Jones, E.; Voudouris, P.; Stouraiti, C.; Moritz, R.; Mavrogonatos, C.; Mitropoulos, P. A new occurrence of terrestrial native iron in the Earth's surface: The Ilia thermogenic travertine case, nrthwestern Euboea, Greece. Geosciences 2018, 8, 287. [CrossRef]

35. Chamberlain, A.; McLeod, C.R.; Traill, R.J.; Lachance, G.R. Native metals in the Muskox intrusion. Can. J. Earth Sci. 1965, 2, 188-215. [CrossRef]

36. Harding, D.J.; Bird, J.M.; Bassett, W.A. The origin of awaruite and native iron in the Josephine peridotite, SW Oregon and NW California. EOS Trans. Am. Geophys. Union 1982, 63, 455.

37. Borisenko, L.F.; Begizov, V.D.; Kurilenko, N.M. Native iron in ore-olivinites of Kachkanarskii massif. Dokl. Akad. Nauk SSSR 1982, 264, 947-950.

38. Rossetti, P.; Zucchetti, S. Occurrence of native iron, Fe-Co and Ni-Fe alloys in the serpentinite from theBalangero asbestos mine (Western Italian Alps). Ofioliti 1988, 13, 43-56.

39. Sakai, R.; Kuroda, Y. Native iron and the associated minerals from the ultramafic masses in the Sanbagawabelt, central Japan. J. Jpn. Assoc. Min. 1983, 78, 467-478.

40. Bai, Y.; Su, B.-X.; Chen, C.; Yang, S.-H.; Liang, Z.; Xiao, Y.; Qin, K.-Z.; Malaviarachchi, S.P.K. Base metal mineral segregation and Fe-Mg exchange inducing extreme compositions of olivine and chromite from the Xiadong Alaskan-type complex in the southern part of the Central Asian Orogenic Belt. Ore Geol. Rev. 2017, 90, 184-192. [CrossRef]

41. Ivan, P.; Jaros, J.; Kratochvil, M.; Reichwalder, P.; Rojkovic, I.; Spisiak, J.; Turanova, L. Ultramafic Rocks of the Western Carpathians, Czechoslovakia; Geologiscky Ustav Dionyza Stura: Bratislava, Slovakia, 1985; 258p.

42. Ploshko, V.V. Listvenitization and carbonatization at terminal stages of Urushten igneous complex, North Caucasus. Int. Geol. Rev. 1963, 7, 446-463. [CrossRef]

43. Menzel, M.D.; Garrido, C.J.; López Sánchez-Vizcaíno, V.; Marchesi, C.; Hidas, K.; Escayola, M.P.; Delgado Huertas, A. Carbonation of mantle peridotite by $\mathrm{CO}_{2}$-rich fluids: The formation of listvenites in the Advocate ophiolite complex (Newfoundland, Canada). Lithos 2018, 323, 238-261. [CrossRef]

44. Beinlich, A.; Plümper, O.; Hövelmann, J.; Austrheim, H.; Jamtveit, B. Massive serpentinite carbonation at Linnajavri, N-Norway. Terra Nov. 2012, 24, 446-455. [CrossRef] 
45. Falk, E.S.; Kelemen, P.B. Geochemistry and petrology of listvenite in the Samail ophiolite, Sultanate of Oman: Complete carbonation of peridotite during ophiolite emplacement. Geochim. Cosmochim. Acta 2015, 160, 70-90. [CrossRef]

46. Hansen, L.D.; Dipple, G.M.; Gordon, T.M.; Kellett, D.A. Carbonated serpentinite (listwanite) at Atlin, British Columbia: A geological analogue to carbon dioxide sequestration. Can. Mineral. 2005, 43, 225-239. [CrossRef]

47. Boskabadi, A.; Pitcairn, I.K.; Broman, C.; Boyce, A.; Teagle, D.A.H.; Cooper, M.J.; Azer, M.K.; Stern, R.J.; Mohamed, F.H.; Majka, J. Carbonate alteration of ophiolitic rocks in the Arabian-Nubian Shield of Egypt: Sources and compositions of the carbonating fluid and implications for the formation of Au deposits. Int. Geol. Rev. 2017, 59, 391-419. [CrossRef]

48. Halls, C.; Zhao, R. Listvenite and related rocks: Perspectives on terminology and mineralogy with reference to an occurrence at Cregganbaun, Co. Mayo, Republic of Ireland. Miner. Depos. 1995, 30, 303-313. [CrossRef]

49. Tsikouras, B.; Karipi, S.; Grammatikopoulos, T.A.; Hatzipanagiotou, K. Listwaenite evolution in the ophiolite melange of Iti Mountain (continental Central Greece). Eur. J. Mineral. 2006, 18, 243-255. [CrossRef]

50. Haas, J.R.; Shock, E.L.; Sassani, D.C. Rare earth elements in hydrothermal systems: Estimates of standard partial molal thermodynamic properties of aqueous complexes of the rare earth elements at high pressures and temperatures. Geochim. Cosmochim. Acta 1995, 59, 4329-4350. [CrossRef]

Publisher's Note: MDPI stays neutral with regard to jurisdictional claims in published maps and institutional affiliations. 